\title{
Measurements and modeling of Alfvén eigenmode induced fast ion transport and loss in DIII-D and ASDEX Upgrade ${ }^{\text {a) }}$
}

\author{
M. A. Van Zeeland, ${ }^{1, b)}$ W. W. Heidbrink, ${ }^{2}$ R. K. Fisher, ${ }^{1}$ M. García Muñoz, ${ }^{3}$ G. J. Kramer, ${ }^{4}$ \\ D. C. Pace, ${ }^{2}$ R. B. White, ${ }^{4}$ S. Aekaeslompolo, ${ }^{5}$ M. E. Austin, ${ }^{6}$ J. E. Boom, ${ }^{7}$ I. G. J. Classen, ${ }^{7}$ \\ S. da Graça, ${ }^{8}$ B. Geiger, ${ }^{3}$ M. Gorelenkova, ${ }^{4}$ N. N. Gorelenkov, ${ }^{4}$ A. W. Hyatt, ${ }^{1}$ N. Luhmann, ${ }^{9}$ \\ M. Maraschek, ${ }^{3}$ G. R. McKee,${ }^{10}$ R. A. Moyer, ${ }^{11}$ C. M. Muscatello, ${ }^{2}$ R. Nazikian, ${ }^{4}$ H. Park, ${ }^{12}$ \\ S. Sharapov, ${ }^{13}$ W. Suttrop, ${ }^{3}$ G. Tardini, ${ }^{3}$ B. J. Tobias, ${ }^{9}$ Y. B. Zhu, ${ }^{2}$ and \\ DIII-D and ASDEX Upgrade Teams \\ ${ }^{1}$ General Atomics, P.O. Box 85608 San Diego, California 92186-5608, USA \\ ${ }^{2}$ University of California-Irvine, University Dr., Irvine, California 92697-4575, USA \\ ${ }^{3}$ Max-Planck-Institut fur Plasmaphysik, EURATOM Association, Boltzmannstr. 2, D-85748 Garching, \\ Germany \\ ${ }^{4}$ Princeton Plasma Physics Laboratory, P.O. Box 451, Princeton, New Jersey 08543, USA \\ ${ }^{5}$ Aalto University, FI-02015 TKK, Finland \\ ${ }^{6}$ University of Texas-Austin, 2100 San Jacinto Blvd, Austin, Texas 78712-1047, USA \\ ${ }^{7}$ FOM-Institute for Plasma Physics Rijnhuizen, Association EURATOM-FOM, Nieuwegein, The Netherlands \\ ${ }^{8}$ Associacao EUATOM/IST, Instituto de Plasmas e Fusao Nuclear-Laboratrio Associado, Instituto Superior \\ Tecnico, Lisboa, Portugal \\ ${ }^{9}$ University of California-Davis, 347 Memorial Un, Davis, California 95616, USA \\ ${ }^{10}$ University of Wisconsin-Madison, 1500 Engineering Dr., Madison, Wisconsin 53706, USA \\ ${ }^{11}$ University of California-San Diego, 9500 Gilman Dr., La Jolla, California 92093, USA \\ ${ }^{12}$ POSTECH, Pohang, Gyeungbuk, 790-784, Korea \\ ${ }^{13}$ Euratom/CCFE, Culham Centre for Fusion Energy, Abingdon, United Kingdom
}

(Received 13 December 2010; accepted 2 March 2011; published online 16 May 2011)

\begin{abstract}
Neutral beam injection into reversed magnetic shear DIII-D and ASDEX Upgrade plasmas produces a variety of Alfvénic activity including toroidicity-induced Alfvén eigenmodes and reversed shear Alfvén eigenmodes (RSAEs). These modes are studied during the discharge current ramp phase when incomplete current penetration results in a high central safety factor and increased drive due to multiple higher order resonances. Scans of injected $80 \mathrm{keV}$ neutral beam power on DIII-D showed a transition from classical to AE dominated fast ion transport and, as previously found, discharges with strong AE activity exhibit a deficit in neutron emission relative to classical predictions. By keeping beam power constant and delaying injection during the current ramp, AE activity was reduced or eliminated and a significant improvement in fast ion confinement observed. Similarly, experiments in ASDEX Upgrade using early $60 \mathrm{keV}$ neutral beam injection drove multiple unstable RSAEs. Periods of strong RSAE activity are accompanied by a large (peak $\delta S_{n} / S_{n} \approx 60 \%$ ) neutron deficit. Losses of beam ions modulated at AE frequencies were observed using large bandwidth energy and pitch resolving fast ion loss scintillator detectors and clearly identify their role in the process. Modeling of DIII-D loss measurements using guiding center following codes to track particles in the presence of ideal magnetohydrodynamic (MHD) calculated AE structures (validated by comparison to experiment) is able to reproduce the dominant energy, pitch, and temporal evolution of these losses. While loss of both co and counter current fast ions occurs, simulations show that the dominant loss mechanism observed is the mode induced transition of counter-passing fast ions to lost trapped orbits. Modeling also reproduces a coherent signature of $\mathrm{AE}$ induced losses and it was found that these coherent losses scale proportionally with the amplitude; an additional incoherent contribution scales quadratically with the mode amplitude. (C) 2011 American Institute of Physics. [doi:10.1063/1.3574663]
\end{abstract}

\section{INTRODUCTION}

Alfvén eigenmodes are routinely observed in present tokamaks ${ }^{1,2}$ and are predicted to be unstable in ITER. ${ }^{3} \mathrm{~A}$ consistent finding among current tokamaks is the ability of these modes to cause enhanced transport of fast ions, ${ }^{4-7}$ resulting in reductions in fusion performance as well as pos-

\footnotetext{
a)Paper TI2 1, Bull. Am. Phys. Soc. 55, 288 (2010).

${ }^{b}$ Invited speaker. Electronic mail: vanzeeland@fusion.gat.com.
}

sible damage to first-wall components. ${ }^{8}$ Because Alfvén eigenmodes (AEs) may interact with both the alpha particles, which are required to maintain fusion burn, as well as high energy beam ions that are critical for heating, momentum input, and current drive, it is obvious that understanding the properties of AEs through modeling and experimental observation is essential in order to have confidence in predictions for their impact on ITER and future devices.

Neutral beam injection into reversed magnetic shear plasmas produces a variety of Alfvénic activity including 
toroidicity-induced Alfvén eigenmodes (TAEs), ${ }^{1}$ reversed shear Alfvén eigenmodes (RSAEs), ${ }^{9,10}$ and linearly coupled RSAEs and TAEs. ${ }^{11-15}$ These modes are studied during the discharge current ramp phase when incomplete current penetration results in a high central safety factor and a strong drive due to multiple higher order resonances. ${ }^{16}$ Historically, much effort has been focused on measurements and modeling of eigenmode structure ${ }^{13,17}$ as well as understanding the impact they have on confined fast ions in DIII-D. ${ }^{4,21}$ This paper expands on those results by detailing a similar impact on confined fast ions in ASDEX Upgrade discharges as well as presenting measurements of lost fast ions and modeling of these measurements. DIII-D lost fast ion measurements are made using a newly installed large bandwidth pitch angle and energy resolving fast ion loss detector (FILD) ${ }^{22}$ similar to the detector currently in operation on ASDEX Upgrade (AUG) ${ }^{23}$ The large bandwidth of these detectors allows resolution of typical AE frequencies and an unambiguous identification of the modes responsible for fast ion loss. The pitch angle and energy resolution are the keys to identify the underlying physics and the details of the wave particle interactions.

This paper is organized as follows. In Sec. II A, DIII-D neutral beam power scan and injection timing scan experiments are presented to give the reader an idea of the global impact AEs have on the fast ion population (using neutron emission and stored energy as a proxy). During periods of strong AE activity, enhanced fast ion transport can cause up to $\mathrm{a} \approx 50 \%$ reduction in neutron emission relative to classical predictions. In Sec. II B, beam driven Alfvén eigenmode ASDEX Upgrade experimental results are shown, where a similar impact on the fast ion population was found. In Sec. III A, measurements of AE induced fast ion loss on DIII-D are presented where it is shown that losses were peaked near the injection energy and occupy a relatively narrow range of pitch angles (width $\approx 5^{\circ}$ ) Modeling of the pitch angle and energy as well as the primary loss mechanism are given in Sec. III B, where it is shown that the dominant loss mechanism observed is the mode induced transition of counterpassing fast ions to lost trapped orbits. Section III C describes modeling which addresses the details of the coherent loss specifically, including the role of resonances and the scaling of coherent losses with amplitude.

\section{IMPACT OF ALFVÉN EIGENMODES ON CONFINED FAST IONS}

\section{A. DIII-D measurements}

Experiments examining the global impact of AEs on the fast ion population for a range of amplitudes were carried out on DIII-D. These experiments utilized two techniques to alter the AE drive and/or stability. The first was a scan of injected beam power and the second was a change in the timing of the initial beam injection during the current ramp. Detailed stability analysis of the modes will be the subject of a future publication; the work presented here focuses primarily on the impact these modes have on the fast ion population.

By scanning injected beam power, the drive for the modes is reduced and at some point becomes lower than the
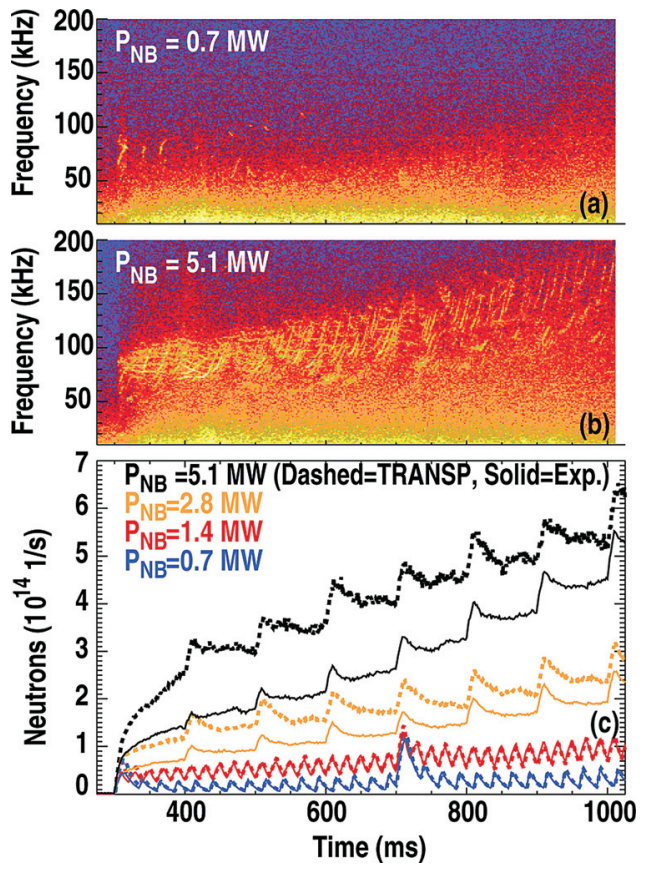

FIG. 1. (Color) Crosspower spectrum of vertical and radial $\mathrm{CO}_{2}$ interferometer chords in (a) 132701 with $P_{N B} \approx 0.7 \mathrm{MW}$ and (b) 132702 with $P_{N B} \approx 5.1$ MW. (c) Measured (solid) and classical TRANSP predicted (dashed) neutron emission for discharges 132702 (black), 132698 (yellow), 132700 (red), and 132701 (blue) with $P_{\mathrm{NB}}=5.1,2.8,1.4$, and $0.7 \mathrm{MW}$, respectively.

combined damping mechanisms. DIII-D's $80 \mathrm{keV}$ neutral beam systems each have a dc injected power of approximately 2.5 MW. To achieve time averaged powers below this, at full voltage, beam modulation is necessary. Figure 1 shows results from a power scan for approximate injected powers of $P_{\mathrm{NB}}=0.7,1.4,2.8$, and $5.1 \mathrm{MW}$ (averaged over several modulation cycles). For reference, typical DIII-D Alfvén eigenmode studies are carried out with the equivalent of $\approx 5 \mathrm{MW}$ injected power during the current ramp phase, and previous Alfvén eigenmode experiments with $P_{\mathrm{NB}}$ as low as 2.3 MW did not show classical neutron emission, ${ }^{4}$ prompting the question whether classical behavior is ever observed this early in the discharge. Crosspower spectra of vertical and radial viewing $\mathrm{CO}_{2}$ interferometer chords ${ }^{17,24}$ are shown in Figs. 1(a) and 1(b) for the $P_{\mathrm{NB}}=0.7$ and 5.1 MW cases, respectively. With the exception of broadband turbulence and a few short occurrences of modes near $f=80-100 \mathrm{kHz}$, the low beam power case is essentially free from $\mathrm{AE}$ activity, whereas the high beam power case clearly has a plethora of AE activity that includes RSAEs and TAEs between 50 and $200 \mathrm{kHz}$. Detailed modeling of these modes has been carried out in several publications where the structure, ${ }^{11-13}$ stability, ${ }^{16}$ and impact on fast ions ${ }^{4,18}$ were investigated.

The impact of these modes on the confined fast ion population is quantified in Fig. 1(c) where comparison of the measured neutron rate (solid) to classical TRANSP (Ref. 25) predictions (dashed) is shown for the various injection powers. For these discharges, the primary neutron production mechanism is through beam-plasma reactions. A neutron deficit relative to classical predictions is a sensitive indicator 
of fast ion redistribution or loss. With on-axis beam deposition, as in these plasmas, the predicted fast ion density is peaked on axis. Increased fast ion transport moves ions to larger radii where density and temperature are lower. Decreased temperature causes the ions to slow down faster causing an effectively reduced fast ion population for a given power. Typically reduced density at larger radii offers less fusion targets, however, in a steady state/relatively constant transport case, the effect of the reduced density is compensated by a corresponding increase in the slowing down time due to reduced density.

In these $B_{T}=2 \mathrm{~T}$ discharges, neutral beam injection begins at $t=300 \mathrm{~ms}$ and continues while the current is ramping up at a constant rate of $0.8 \mathrm{MA} / \mathrm{s}$ until reaching approximately 1.2 MA at $t=1000 \mathrm{~ms}$. Since the neutron emission is weighted toward highest energies, the rapid beam modulation $(\approx 20-30 \mathrm{~ms})$ in the two lowest beam power cases causes the observed sawtooth-like appearance. The occasional modulation of the two higher power cases is due to the blip of an additional beam every $100 \mathrm{~ms}$ for diagnostic purposes. It is noted that, because of uncertainty in the absolute neutron calibration, the "experimental" neutron rate in this figure has been adjusted by a constant normalization factor that gives agreement with the average TRANSP magnitudes for the lowest power case over the interval $t=300$ $1100 \mathrm{~ms}$. Similar low-power beam heated discharges were carried out when a neutron calibration was available, indeed show classical neutron emission during AE free periods of the current ramp. ${ }^{4}$ In Fig. 1(c), the shape of the measured neutron emission temporal behavior is essentially classical for the two lowest beam power cases, then diverges significantly from classical predictions in both shape and magnitude for the two higher beam power cases. Also, the ratio of the two lowest power cases is essentially the same as that from classical predictions. Both the $P_{\mathrm{NB}}=2.8$ and 5.1 MW cases have qualitatively similar mode activity in that both RSAEs and TAEs are present as in Fig. 1(b); however, the average mode amplitude is larger in the $P_{\mathrm{NB}}=5.1 \mathrm{MW}$ discharge where up to $\approx 40 \%$ neutron deficits are observed.

In addition to scanning beam power and reducing the Alfvén eigenmode drive directly, it was observed that delaying beam injection by $240 \mathrm{~ms}$, while keeping the current ramp rate constant $(\approx 0.8 \mathrm{MA} / \mathrm{s})$, causes the level of Alfvén eigenmode activity to be greatly reduced and in some cases made completely stable for the same injected power. This result is shown in Fig. 2, where Figs. 2(a) and 2(b) are spectrograms of the early $(t=300 \mathrm{~ms})$ and later $(t=520 \mathrm{~ms})$ injection, respectively. By delaying beam injection during the current ramp phase, the plasma remains cooler in the core and the current is allowed to penetrate faster, causing a more weakly reversed shear profile and lower $q_{\min }$ for a given time. In turn, the lower central $q$-values reduce the drive of the modes that are primarily interacting with the fast ions through higher order resonances. ${ }^{16} \mathrm{~A}$ detailed analysis of the stability of these modes due to delayed beam injection is underway and will be the subject of a future publication.

Both the measured and classically predicted neutron emissions are given for the "early" and "late" injection cases in Figs. 2(c) and 2(d), respectively. As expected, the more
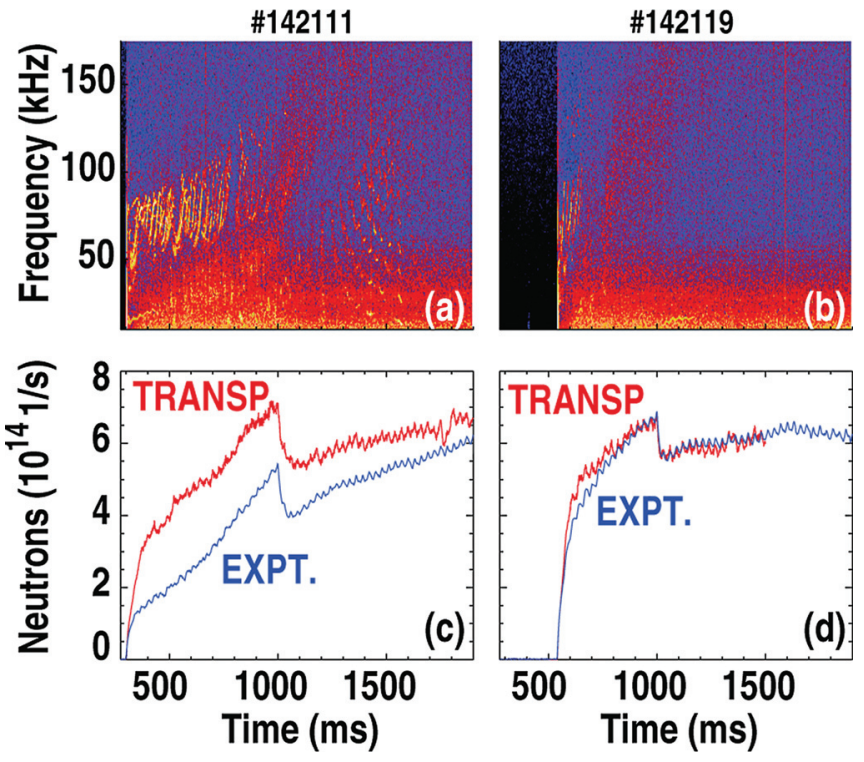

FIG. 2. (Color online) Crosspower spectrum of vertical and radial $\mathrm{CO}_{2}$ interferometer chords in (a) 142111 with $P_{N B} \approx 6.8 \mathrm{MW}$ beginning at $t=300$ ms and (b) 142119 with $P_{N B} \approx 6.8 \mathrm{MW}$ beginning at $t=540 \mathrm{~ms}$. Measured (blue) and classical TRANSP predicted (red) neutron emission for discharge (c) 142111 and (d) 142119 .

typical early injection case, with multiple unstable modes, has much reduced neutron emission relative to classical predictions. The discharges in Fig. 2 were not carried out on a day with low beam power (as in Fig. 1), so the approximate neutron calibration was obtained by scaling experimental measurements to TRANSP predictions for the delayed injection case (142119) over the interval $t=1400-1500 \mathrm{~ms}$ during which no magnetohydrodynamic (MHD) or AEs were observed.

A quantitative estimate of the level of fast ion diffusion present in discharge 142111 [Figs. 2(a) and 2(c)] is determined through a series of TRANSP runs with varying levels of fast ion diffusivity. The fast ion diffusivities used $\left(D_{B}=0\right.$, $0.5,1.0,2.5$, and $5.0 \mathrm{~m}^{2} / \mathrm{s}$ ) were independent of position, pitch, and energy. The expected neutron emission for $D_{B}=0,1.0$, and $5.0 \mathrm{~m}^{2}$ is shown in Fig. 3(a) along with the measured neutron emission. From this figure, one can see that the neutron emission is initially consistent with a fast ion diffusivity of $\approx 5 \mathrm{~m}^{2} / \mathrm{s}$. Then, as the discharge evolves, the neutron emission becomes near classical by $t=1900 \mathrm{~ms}$. The assumption of a uniform pitch/energy independent diffusivity is admittedly crude and only serves to put the measured transport on some type of quantitative scale. In reality, as will be addressed in Sec. III C, each individual mode interacts with the fast ion population along well defined resonances. However, as more and more modes are considered that span the plasma with multiple overlapping resonances, this approximation may become a more accurate representation of the situation.

The multiple TRANSP runs can be used to obtain an effective diffusivity as a function of time by linear interpolation of the measured neutron rates between the simulated values for the various $D_{B}$. The time evolution of the resultant effective fast ion diffusivity is given in Fig. 3(b). When a 

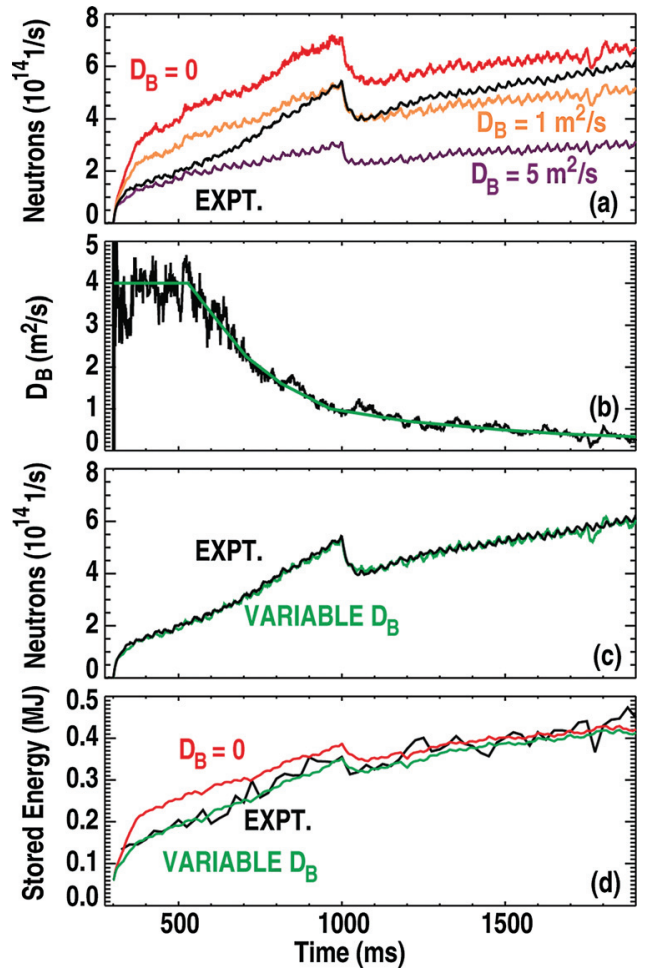

FIG. 3. (Color) Discharge 142111. (a) Measured (black) and TRANSP predicted neutron emission with $D_{B}=0 \mathrm{~m}^{2} / \mathrm{s}$ (red), $1 \mathrm{~m}^{2} / \mathrm{s}$ (yellow), $5 \mathrm{~m}^{2} / \mathrm{s}$ (purple). (b) Effective time dependent diffusivity obtained through linear interpolation of several constant diffusivity TRANSP runs (black) and smoothed (green). (c) Measured (black) and TRANSP calculated (green) neutron emission, where TRANSP calculation used variable fast ion diffusivity from (b). (d) Stored energy predicted by TRANSP with $D_{B}=0$ (red) and variable $D_{B}$ (green) compared to measured (black).

separate TRANSP run is carried out using this effective diffusivity, the calculated neutron emission closely matches experiment [Fig. 3(c)].

Further corroborating this approach and the resultant large impact on the confined fast ion population, the TRANSP calculated stored energy for the $D_{B}=0$ and variable $D_{B}$ cases along with experiment are shown in Fig. 3(d). From this figure, one finds that while both the variable and zero diffusion cases appear to be within the experimental noise after $t \approx 1200 \mathrm{~ms}$, the variable $D_{B}$ TRANSP run closely matches the measured stored energy early in the discharge when the diffusion is large. The pronounced difference at earlier times is due to two reasons, the first is simply that the diffusivity is larger. The second compounding factor is that early in the discharge, the beam ion pressure is a larger fraction of the total pressure.

\section{B. ASDEX Upgrade measurements}

Recently, neutral beam driven Alfvén eigenmode experiments, similar to those described in Sec. II A, were also carried out on ASDEX Upgrade. These experiments are described in detail in Refs. 14 and 15, however, two of the primary goals were verification of significant Alfvén eigenmode induced transport as well as establishing conditions for future collaborative beam driven Alfvén eigen-

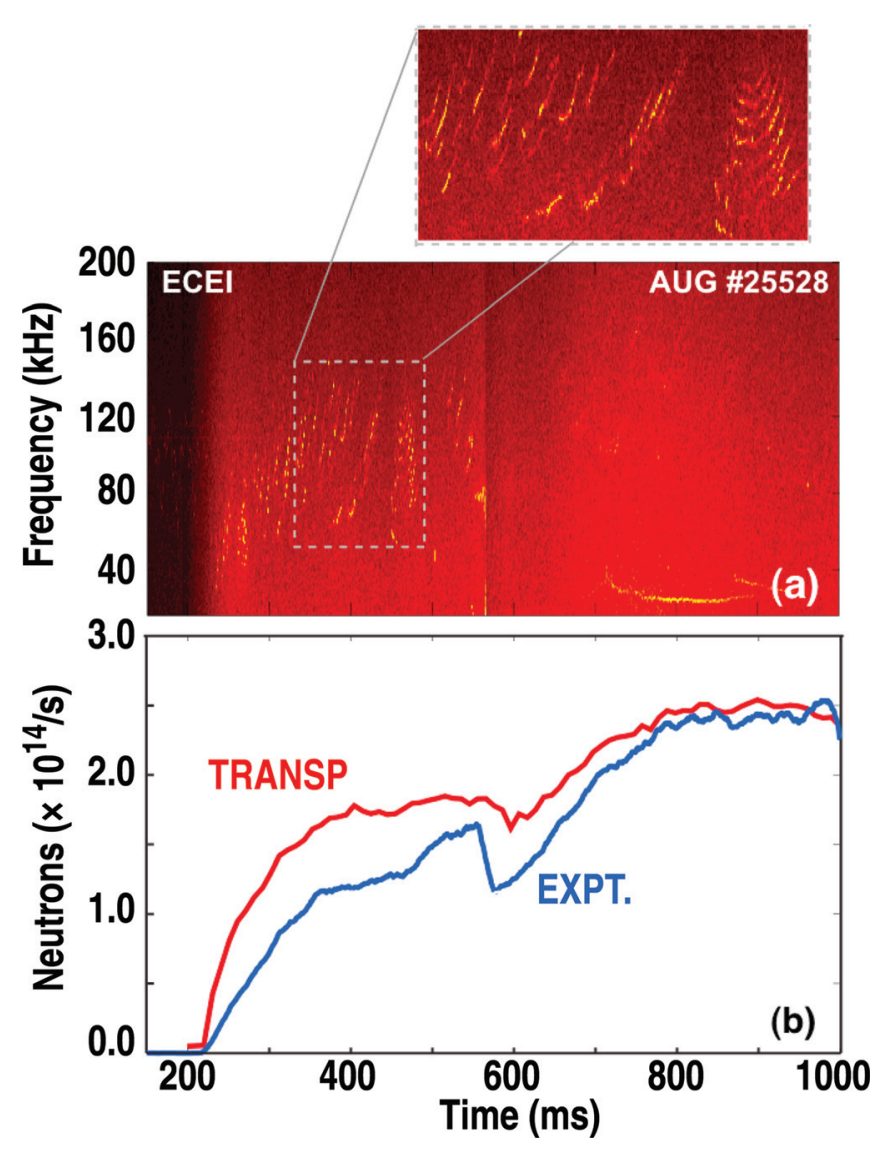

FIG. 4. (Color online) AUG Discharge 25528. (a) Crosspower spectrogram of ECEI data with expanded region shown above to accentuate the multitude of RSAEs present in discharge. (b) Measured neutron emission (blue) and classical TRANSP predicted neutron emission (red). Sixty kiloelectronvolts neutral beam injection begins at $t=200 \mathrm{~ms}$.

mode studies. As with the DIII-D Alfvén eigenmode studies, these experiments used early beam injection during the current ramp phase to create reversed magnetic shear and keep $q_{\min }$ elevated-key elements for increasing the drive of AEs at high toroidal field through higher order resonances. ${ }^{16}$

Figure 4(a) shows an electron cyclotron emission imaging (ECEI) spectrogram obtained during a discharge with 60 $\mathrm{keV}$ beam injection beginning at $t=200 \mathrm{~ms}$ during the current ramp of a $B_{T}=2.5 \mathrm{~T}$ discharge. ${ }^{14,15,26}$ In this discharge, a multitude of RSAEs were driven unstable, highlighted in the expanded portion of Fig. 4(a). Figure 4(b) shows the classical neutron emission calculated by TRANSP as well as that measured experimentally; clearly, early during the discharge when RSAE activity is more pronounced, there is a significant deficit in the neutron emission. As the RSAE activity weakens, the neutron deficit is reduced until $\approx 800 \mathrm{~ms}$ when the neutron emission is essentially classical. The same beam driven Alfvén eigenmode experiments on ASDEX Upgrade also revealed for the first time pitch angle and energy resolved coherent losses of beam ions due to Alfvén eigenmodes, ${ }^{14,15}$ as well as, using the newly installed AUG fast ion $\mathrm{D}_{\alpha}$ diagnostic, ${ }^{27}$ a large central flattening of the fast ion profile concomitant with the excitation of several RSAEs at a $q_{\text {min }}$ integer crossing. ${ }^{14,15}$ 

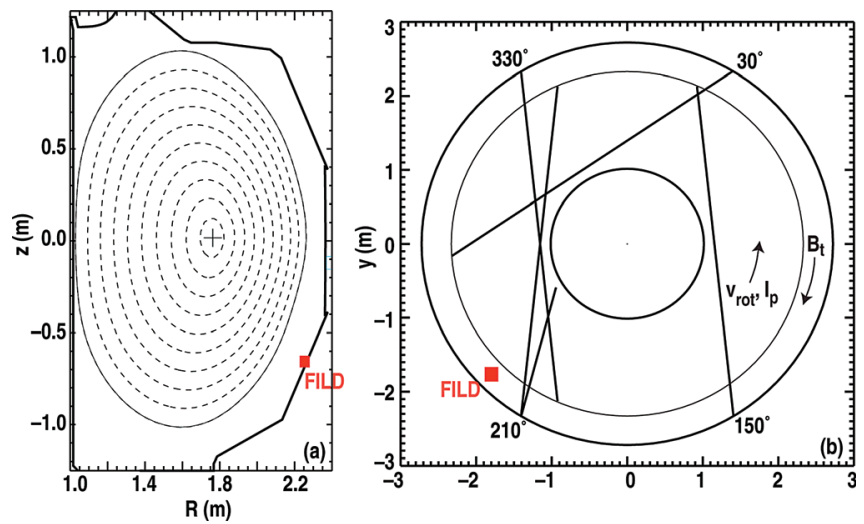

FIG. 5. (Color online) (a) Cross-section of DIII-D inner wall with FILD location overlayed. Dashed contours are equally spaced contours of $\rho$ for discharge 142111 at $t=525 \mathrm{~ms}$. (b) Top view of DIII-D showing toroidal location of FILD as well as beam line trajectories that were used during times of interest. $150^{\circ}, 30^{\circ}$, and $330^{\circ}$ beamlines are in the direction of plasma current and those from $210^{\circ}$ are counter current. Direction of toroidal rotation $\left(V_{\text {rot }}\right)$, plasma current $\left(I_{p}\right)$, and toroidal field $\left(B_{t}\right)$ is shown.

\section{FAST ION LOSS DUE TO ALFVÉN EIGENMODES}

Because measurements such as neutron emission, stored energy, and fast ion d-alpha (FIDA) (Ref. 18) average over a significant fraction of the distribution function and often lack mode-frequency timescale response, they are relatively indirect methods for inferring the impact AEs have on fast ion confinement. Recently, a large bandwidth pitch angle and energy resolving fast ion loss detector, capable of directly measuring losses at AE frequencies, was installed and commissioned on DIII-D. ${ }^{22,28-30}$ This section describes initial measurements of Alfvén eigenmode induced fast ion losses as well as modeling of these losses.

\section{A. Measurements}

The primary discharge discussed in relation to FILD measurements is 142111 [Figs. 2(a), 2(c), and 3], which contains a variety of coherent mode activity including TAEs,

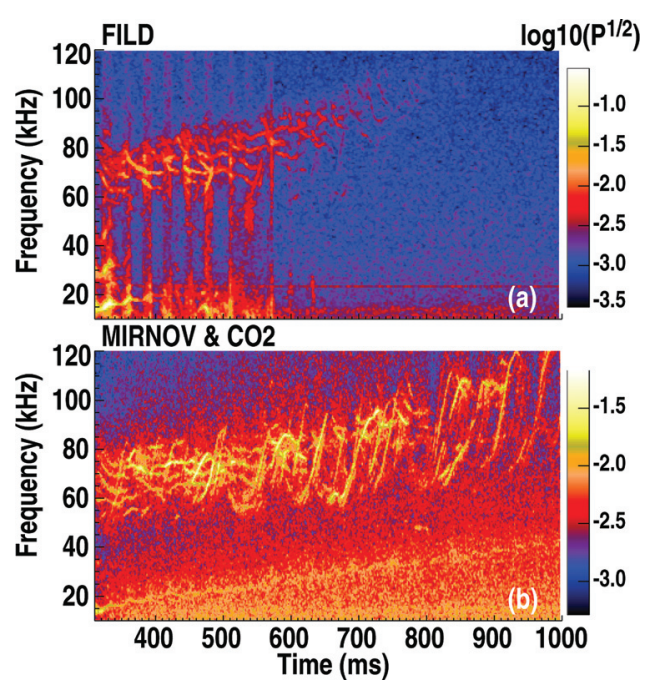

FIG. 6. (Color online) Discharge 142111. (a) Spectrogram FILD data. (b) Crosspower spectrum of Mirnov (magnetic fluctuations) and $\mathrm{CO}_{2}$ interferometer data.

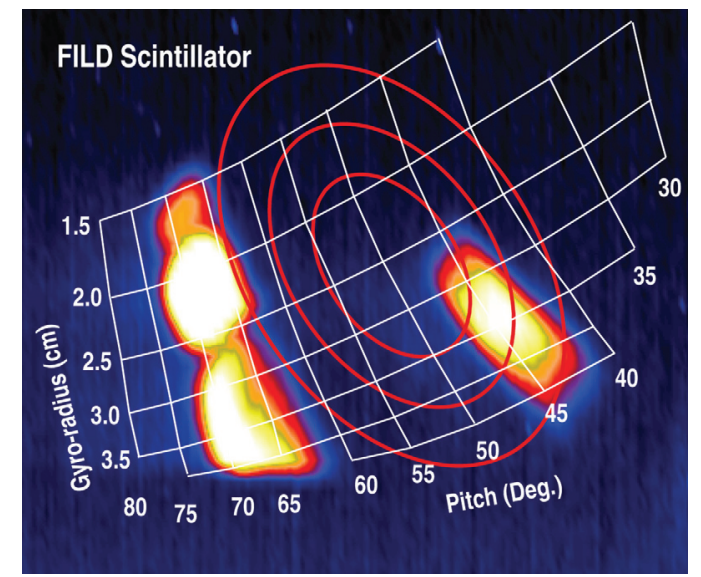

FIG. 7. (Color online) FILD scintillator image, discharge 142111 at $t=525$ $\mathrm{ms}$, with $500 \mu$ s exposure. Overlayed red contours represent 25, 50, and $75 \%$ peak amplitude contours of viewing optic for large bandwidth measurements.

RSAEs, energetic particle induced geodesic acoustic mode (EGAMs), ${ }^{31}$ and beta induced Alfvén acoustic eigenmode (BAAEs) $^{32}$ and has up to $50 \%$ neutron deficit relative to classical TRANSP predictions. The FILD location relative to the DIII-D vessel and plasma is shown in Fig. 5 as well as the centerlines of neutral beams that were used during the times of interest. A spectrogram from the central portion of the FILD scintillator during the current ramp phase of this discharge is shown in Fig. 6(a) along with a crosspower spectrum of magnetic and $\mathrm{CO}_{2}$ interferometry data. The magnetic and $\mathrm{CO}_{2}$ crosspower spectrum is given as a global measure of the mode activity present in the discharge. The FILD diagnostic clearly sees losses from TAEs, RSAEs, and the EGAM-the coherent peaks make this unambiguous, which is truly the largest advantage of large bandwidth fast ion loss measurements such as these. TAEs are the series of relatively constant frequency modes above $60 \mathrm{kHz}$, RSAEs are the modes which sweep up into the TAE range and the EGAM (and its harmonics) are below approximately $50 \mathrm{kHz}$ and strongest early in the discharge $(t<400 \mathrm{~ms})$. In comparing the two spectra in Fig. 6, one thing that stands out is the apparent lack of losses due to AEs after $t \approx 800 \mathrm{~ms}$, despite the fact that many such modes are still present in the plasma and of comparable amplitude.

Data from the FILD scintillator are shown in Fig. 7 for $t=525 \mathrm{~ms}$. The white regions indicate the gyroradius and pitch $\left[\chi=v_{\|} / v\right.$, and $\chi_{D}=\cos ^{-1}(\chi)$ in deg] of lost ions striking the detector. The overlayed red contours indicate the approximate region sampled by the fast photodetector used to create the power spectrum in Fig. 6(a). The losses at approximately $\chi_{D}=70^{\circ}$ correspond primarily to prompt losses of full, half, and third energy beam components as described in Ref. 28. The full $80 \mathrm{keV}$ injection energy gyroradius at FILD is approximately $R_{L}=3.7 \mathrm{~cm}$. The losses near $\chi_{D} \approx 45^{\circ}$ and $R_{L} \approx 3.5 \mathrm{~cm}$ do not correspond to prompt losses (as they are not modulated with any beam) and are associated with mode induced loss which can include contributions from both AEs and the lower frequency MHD also observed at this time.

Modeling of these losses, described in the next two sections, will focus on two distinct time ranges in discharge 
142111: $t \approx 525 \mathrm{~ms}$ and $t \approx 725 \mathrm{~ms}$. The time ranges were chosen with several modeling goals in mind: (1) identifying the origin of the losses, (2) identifying expected pitch and energy of the losses at the FILD location, (3) explaining reduction in loss levels as current increases (later time), and (4) reproducing signature of coherent losses in simulation.

\section{B. Modeling-loss to FILD}

The primary theoretical tool used to model fast ion loss in these experiments is the Hamiltonian guiding center code ORBIT. ${ }^{33}$ ORBIT calculates particle trajectories in a tokamak in the presence of input wavefields superimposed on an equilibrium field. In this application, the input eigenmodes are taken from the ideal MHD solver NOVA. ${ }^{34,35}$ ORBIT combined with NOVA calculated Alfvén eigenmodes was recently used to successfully model the impact of AEs on confined fast ions in DIII-D plasmas ${ }^{20,21}$ and has also been used for similar studies in national spherical torus experiment (NSTX). ${ }^{36}$ A similar methodology is employed here except further analysis is required to follow particles to the FILD.

First, equilibrium profiles of density, temperature, impurity density, etc., are fit and used as inputs to calculate the linear eigenmodes of the plasma with NOVA and the expected beam ion deposition using TRANSP. Next, a subset of these eigenmodes is identified from experimental measurements including electron cyclotron emission (ECE), ${ }^{37}$ $\mathrm{ECEI},{ }^{38,39}$ beam emission spectroscopy (BES), ${ }^{40}$ magnetics, and interferometry. By fitting the eigenmode structures to ECE data, a mode amplitude is determined. ${ }^{13,17}$ ORBIT is then used to calculate the transport of the TRANSP calculated beam ion distribution in the presence of the NOVA calculated wavefields. Particles that reach the last closed flux

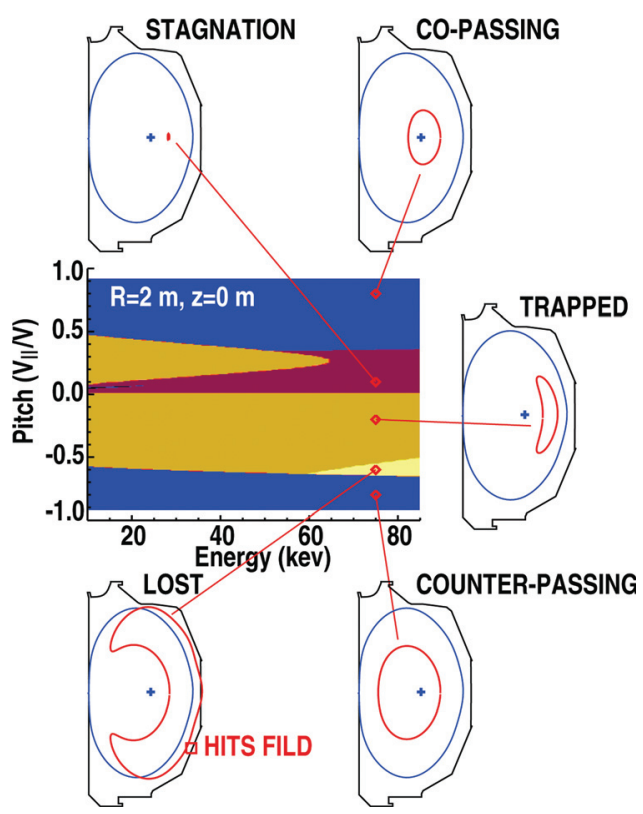

FIG. 8. (Color) Contour of orbit classifications calculated with COM based code for particles initiated at $R=2 \mathrm{~m}$ and $z=0 \mathrm{~m}$ with $E=10-85 \mathrm{keV}$ and $\chi=-0.92$ to +0.92 in discharge 142111 at $t=525 \mathrm{~ms}$. Trajectories of representative orbits at $E=75 \mathrm{keV}$ are indicated in red and LCFS in blue. Lost trajectory intersects FILD. surface (LCFS) in the simulation are identified as lost and a second constants of motion (COM) based code (described in the appendix) follows those particles further to determine which reach the FILD detector. Note, this approach is required with the version of ORBIT being employed and restricts mode amplitudes to zero outside of the LCFS.

A survey of typical orbits in discharge 142111 at $t=525$ ms calculated using the COM based code is shown in Fig. 8. The contour shows regions of different orbit classes for a range of energies and pitch angles launched at $(R, z)=(2,0) \mathrm{m}$. The light-yellow region is perhaps the most important in that it shows the energies and pitch angles which are lost, with an example orbit given that actually hits the FILD diagnostic. The presence of loss boundaries within the plasma, which have orbits intersecting the FILD, is necessary if one expects to be able to model fast ion losses measured at the detector using the approach outlined here. Without such loss regions, particles could be "lost" (in the ORBIT sense of being pushed past the LCFS), however there would be no possibility of them intersecting the FILD detector. Put another way, since the mode amplitude is assumed zero outside of the LCFS and particles are not allowed to re-enter the plasma and interact further with the modes; if the particle is not on a trajectory intersecting FILD when it first reaches the LCFS, there is no possibility for it to reach FILD.

The primary eigenmodes relevant for fast ion loss are identified using multiple diagnostics. Figure 9(a) shows an average crosspower spectrum of adjacent ECE channels for the early modeling time window in which multiple TAEs and RSAEs are clear. Figure 9(b) is the same spectrum except points representing frequencies at which a coherence above the $95 \%$ confidence level between several adjacent

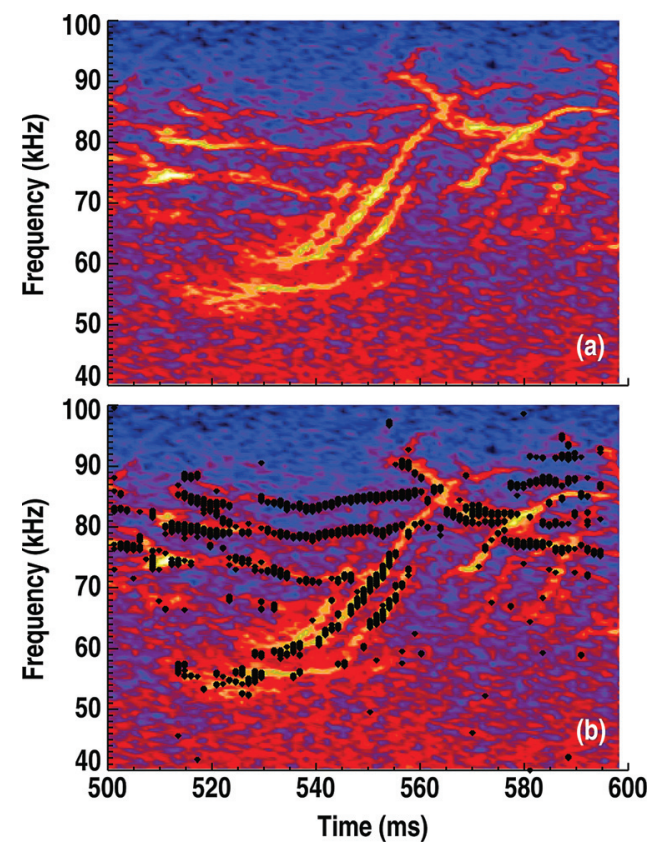

FIG. 9. (Color online) (a) Discharge 142111, average crosspower spectrum of 20 adjacent ECE channel pairs. (b) Same spectrum as panel (a) with overlayed points corresponding to frequencies at which coherence between several adjacent ECE channels and FILD detector is greater than 95\% significance level-these global coherent modes are causing fast ion loss to FILD. 

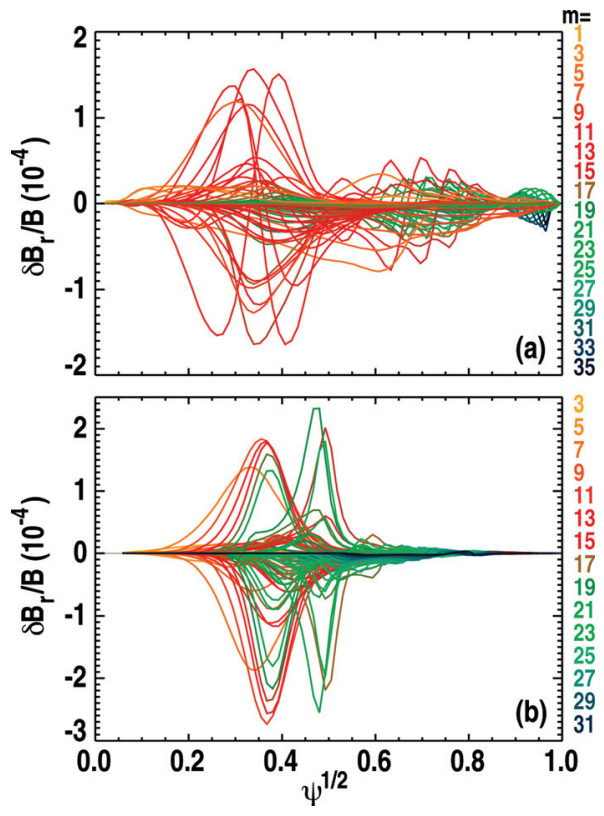

FIG. 10. (Color online) Input radial magnetic field perturbation along outboard midplane used in ORBIT calculations for modeling of (a) early and (b) late time cases. Color coded poloidal mode number in Boozer coordinates of every other harmonic is shown to right of each panel. (a) 109 poloidal harmonics, representing eight individual modes with toroidal mode numbers $n=5,4,3,1,4,3,3,2$ and frequencies $f=75.5370,72.8880$, $69.7870,62.3920,80.4420,73.8470,68.7460,71.6060 \mathrm{kHz}$. (b) 126 poloidal harmonics, representing nine individual modes with toroidal mode numbers $n=2,3,4,5,6,7,5,6,7$ and frequencies $f=71.8170,70.0590,70.8810$, $77.8010,78.3290,80.8200,72.0890,75.6880,76.8200 \mathrm{kHz}$.

ECE channels and the FILD diagnostic are overlayed-these are the global modes causing fast ion loss to FILD. An array of unequally spaced Mirnov coils is then used to identify the toroidal mode number of these modes and the approximate poloidal mode number is extracted from ECEI and BES measurements combined with analytic modeling of RSAE frequency evolution. With this information, the experimental modes are then matched up to NOVA calculated eigenmodes as in Refs. 13, 17 and the full mode structure with experimentally determined amplitudes is obtained for input to ORBIT. In general, NOVA finds several eigenmodes for each toroidal mode number ${ }^{36}$ and identification of mode type is aided by carrying out calculations for a range of $q_{\text {min }}$. When NOVA solutions are found for several values of $q_{\mathrm{min}}$, the distinction between RSAEs and TAEs becomes obvious due to the obtained eigenmode frequency variation lending more confidence that the modes being used are representative of those measured in experiment. The radial structure of the magnetic field perturbation along the outboard midplane for more than 100 poloidal harmonics at each time interval is given in Fig. 10.

The use of NOVA calculated linear eigenmodes assumes that the calculated linear eigenmode structures are an adequate representation of the experimental nonlinearly saturated eigenmodes as well as the fact that these structures do not interact and modify each other significantly (beyond any linear mode coupling already present in the input eigenmodes ${ }^{12}$ ). Several studies have shown that the linear eigenmodes in these type of DIII-D plasmas do in fact repro-
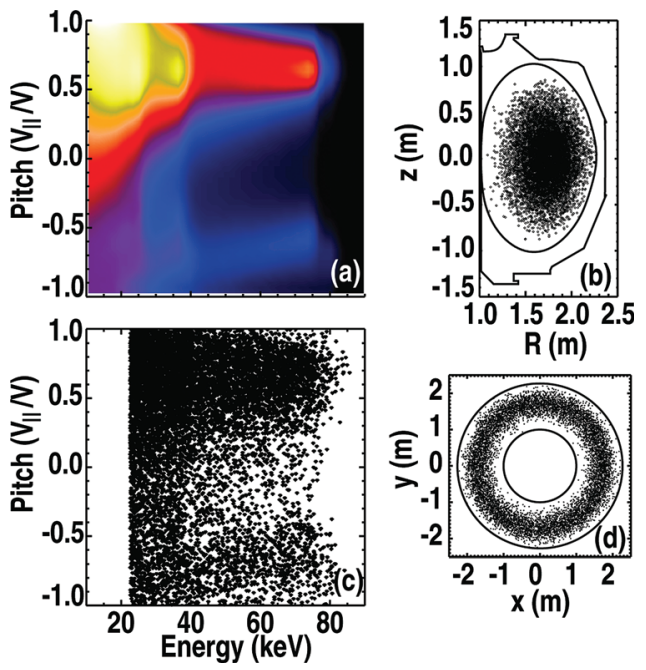

FIG. 11. (Color online) (a) Spatially averaged TRANSP distribution function used for early time case. White is high density, black is zero. Subset of launched particles showing initial (b) poloidal, (c) energy and pitch, (d) toroidal distributions.

duce, remarkably well, many of the features of the experimentally measured structures. ${ }^{12,17,39}$ Fast ion diamagnetic flows appear to modify the relative phase of the various poloidal harmonics slightly, yet still retain the overall eigenmode radial amplitude envelope. ${ }^{39}$ Essentially, by selecting these eigenmodes, we are simply asking the question, can a set of fixed linear eigenmodes with many features of those measured experimentally reproduce the measured fast ion loss.

The initial fast ion distribution function for the transport calculations is obtained from TRANSP calculations and is shown (averaged over the plasma) in Fig. 11(a). The initial positions, pitch, and energy of a subset of the launched particles are shown in Figs. 11(b)-11(d). As can be seen, the particle distribution is centrally peaked and distributed uniformly in the toroidal direction. Additionally, particle energies below $23 \mathrm{keV}$ were not considered (23 keV perpendicular energy corresponds to $\sim 2 \mathrm{~cm}$ Larmor radius at the FILD, below which no losses due to modes were observed). It can also be seen that a significant number of both co $(\chi>0)$ and counter $(\chi<0)$ current going particles was included.

When the eigenmodes from Fig. 10 were allowed to operate on the launched fast ions, losses were observed. Simulations were carried out for $0,0.5,1,2.5$, and $5 \times$ the experimental amplitudes as well as with and without pitch angle scattering. ${ }^{20}$ The fraction of lost particles (crossing the LCFS) for the various runs as a function of time is shown in Figs. 12(a) and 12(b). In all cases (even without modes), some particles are promptly lost. These particles correspond to orbits that are nominally confined but spend a portion of their trajectory outside the LCFS. In all cases with modes, more losses occur than without modes and with scattering included, an additional level of loss occurs due to particles being scattered onto loss orbits. The asymptotic loss rates with and without scattering are given in Fig. 12(c) where it appears that the collisions simply shift the level of losses by 

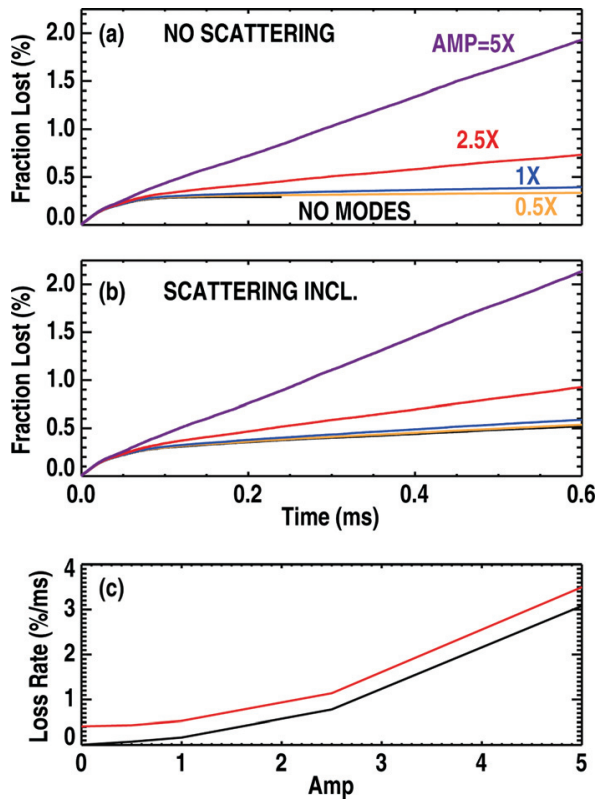

FIG. 12. (Color) Fraction of launched particles lost in early time case ORBIT simulations using modes with $0,0.5,1,2.5$, and $5 \times$ experimental amplitudes and (a) NO pitch angle scattering (b) pitch angle scattering included. (c) Asymptotic loss rate vs mode amplitude scaling factor with (red) and without (black) pitch angle scattering included.

some relatively constant amount. In these simulations, $\sim 8 \%$ of the confined beam ion population is lost through the LCFS in a full energy slowing down (50 ms at mid-radius) period when using experimental amplitudes.

Figure 13 gives details about the pitch and energy of lost particles for the case without pitch angle scattering included. A sample of the launched particles is shown in Fig. 13(a), along with all particles crossing the LCFS (lost) in

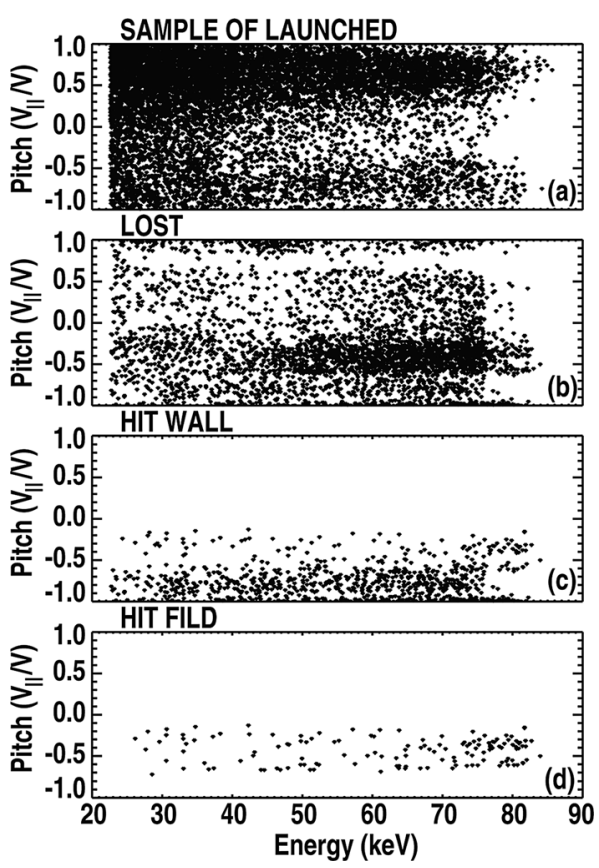

FIG. 13. Pitch and energy distribution of (a) subset of launched particles, (b) all lost particles, (c) all particles intersecting wall, and (d) all particles that intersect FILD $R, z$. Results are for early time run at $\approx 2.5 \times$ experimental amplitude without pitch angle scattering.

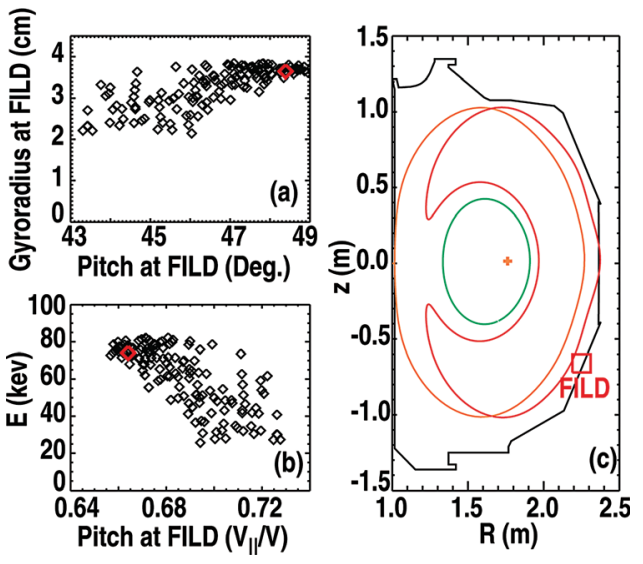

FIG. 14. (Color) (a) Gyroradius and pitch angle of particles hitting FILD shown in Fig. 13(d). (b) Data from panel (a) replotted vs energy and $v_{\|} / v$. (c) Green = unperturbed poloidal trajectory of particle with same initial position, energy, and pitch as lost particle shown as red diamond in panels (a) and (b). Red = unperturbed poloidal trajectory of particle with energy, pitch, and position, where red diamond particle in panels (a) and (b) intersects LCFS in ORBIT simulations. LCFS is shown in orange.

Fig. 13(b), hitting the wall in Fig. 13(c), and those which intersect FILD in Fig. 13(d). The results shown are for the $2.5 \times$ experimental amplitude case as better statistics were obtained for the analysis/figures to follow with negligible changes to the conclusions. In these simulations, both co and counter going fast ions were lost with the majority clearly being initialized on counter going orbits. The particles that hit the wall and FILD [Figs. 13(c) and 13(d)] were initialized solely on counter going orbits. As mentioned, this restriction to counter going orbits is in part a consequence of the technique employed which requires a loss boundary present in the plasma with a trajectory reaching FILD. Coherent losses of particles to FILD have been observed in discharges without counter beam injection, and it is possible that some of the particles initialized on cocurrent orbits, which were lost, could reach FILD if they were allowed to re-enter the plasma and interact with the modes further. Preferential losses of counter current fast ions such as those found here were observed in previous simulations, where in fact, a more selfconsistent treatment of the problem was carried out. ${ }^{41,42}$

All particles that hit the LCFS are followed with the COM based code and when a particle comes within $7.5 \mathrm{~cm}$ of the FILD poloidal position, its pitch and gyroradius are recorded. The collection radius of $7.5 \mathrm{~cm}$ used is larger than the actual collection volume and was chosen to improve the loss statistics. Figure 14(a) gives the gyroradius and pitch of all particles found to strike FILD. For comparison, the energy and pitch as $v_{\|} / v$ are given in Fig. 14(b). The peak of the losses which strike FILD is near the injection energy ( 80 $\mathrm{keV}$ ) and some particles are observed down to the lower energy limit of $23 \mathrm{keV}$. All particles striking FILD are found to be confined to a narrow region in pitch $43^{\circ}-49^{\circ}$ $\left(v_{\|} / v \approx 065-073\right)$. An example of the initial (final) unperturbed orbit for a particle near the peak of those hitting FILD is shown in green (red) in Fig. 14(c). The initial orbit is the poloidal trajectory the particle would have taken with no mode present and the final trajectory is the equivalent for a particle with the position and velocity at the point where the 


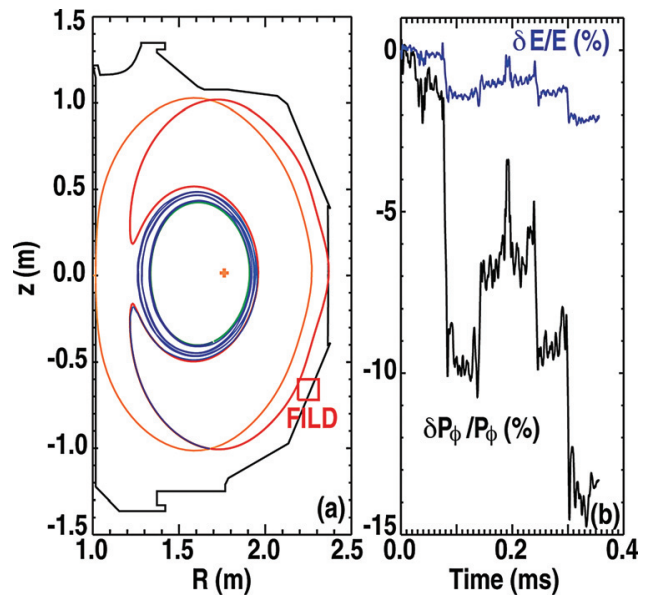

FIG. 15. (Color) (a) ORBIT calculated trajectory of lost particle intersecting FILD (blue). Unperturbed initial (green) and final (red) trajectories from Fig. 14(c) are also shown. LCFS is shown in orange. (b) Energy $(\delta E / E)$ and toroidal canonical angular momentum $\left(\delta P_{\phi} / P_{\phi}\right)$ evolution of lost particle.

lost particle hits the LCFS. This particle, like almost every other particle hitting FILD in the simulations, was initialized on a counter passing orbit (green) which eventually, through interaction with the modes, was transferred to a lost trapped orbit intersecting FILD on its cogoing leg.

The actual path of this lost particle in Fig. 14(c) takes while interacting with the modes is shown in Fig. 15(a). As can be seen from this figure, the particle loses energy and toroidal canonical angular momentum [Fig. 15(b)] as it is pushed to larger minor radius where it finally gets set on a loss orbit. Once on the loss orbit, it very closely follows the unperturbed trajectory shown in red. This type of loss trajectory is the so-called "big last step" and is typical for the particles observed to hit FILD. Losses of alpha particles born on prompt loss orbits such as this were observed using scintilla-
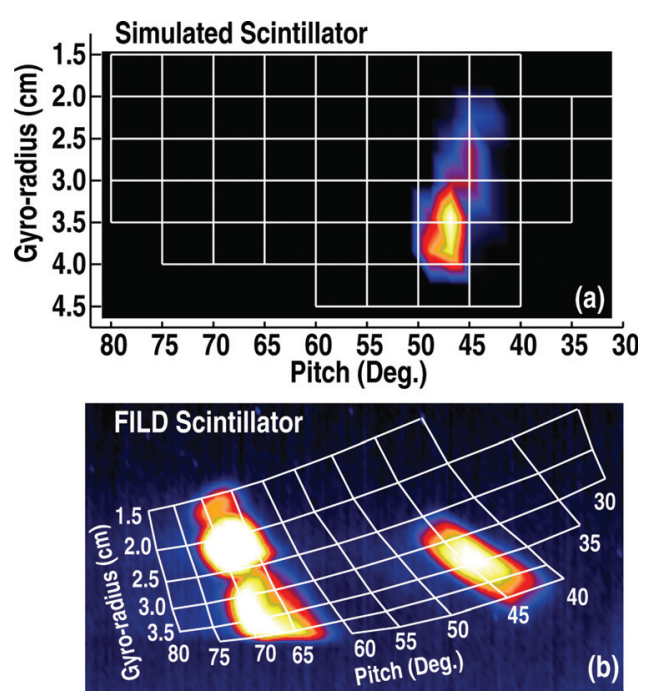

FIG. 16. (Color online) (a) Synthetic scintillator diagnostic constructed by binning particles [shown in Fig 14(a)] in energy and pitch that strike FILD. White represents more particles strikes and black represents zero. (b) FILD scintillator image showing prompt losses at $\chi_{D} \approx 70^{\circ}$. and MHD induced losses centered near $\chi_{D} \approx 45^{\circ}$ and $R_{L} \approx 3.5 \mathrm{~cm}$. Note, prompt losses are intentionally excluded from ORBIT results.

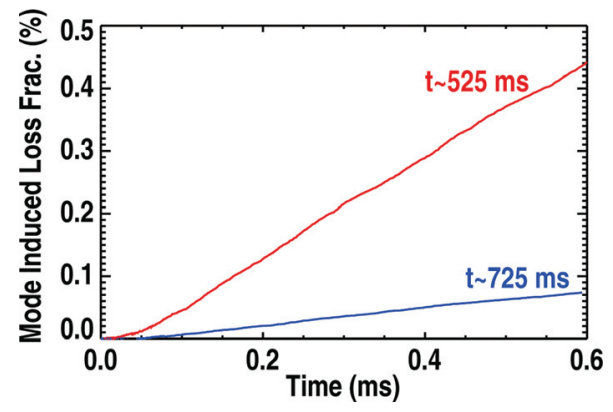

FIG. 17. (Color online) Comparison of the temporal evolution of nonprompt losses in ORBIT simulations for conditions corresponding to the "early" time case $(t \approx 525 \mathrm{~ms})$ and "late" time case $(t \approx 725 \mathrm{~ms})$. Note, comparison is made of the $2.5 \times$ experimental amplitude case.

tor detectors on tokamak fusion test reactor (TFTR) ${ }^{43,44}$ and were also found to be the dominant loss mechanism in historical ORBIT simulations of alpha loss induced by TAE modes. ${ }^{45,46}$ In fact, in the alpha loss study presented in Ref. 46 it was remarked "The statistically most likely loss process is the fluctuation-driven conversion of resonant countergoing circulating alphas near the passing/trapped boundary to large trapped orbits. The conversion occurs because of the large resonant change of energy $\left(E_{\alpha}\right)$ and thus the pitch angle parameter $\mu B / E_{\alpha}$."

Taking the collection of particles which strike FILD, one can construct a simple synthetic loss scintillator diagnostic by histogramming the particles in energy and pitch. The results from application of this technique to the simulation results in Fig. 14(a) are shown in Fig. 16(a) and for comparison, the actual scintillator is shown in Fig. 16(b). The results are that the synthetic scintillator diagnostic reproduces the approximate energy and pitch of the measured loss spot that does not come from prompt beam ion loss.

All discussion of modeling results until now has focused on the early time region near $t \approx 525 \mathrm{~ms}$ in 142111. Another focus of the modeling was to see if simulations could reproduce the decrease in losses observed at later times $t \approx 725 \mathrm{~ms}$. The temporal evolution of the fraction of lost particles for the early time and later time simulations is shown in Fig. 17, where the decrease in total losses for the $t \approx 725 \mathrm{~ms}$ case relative to the $t \approx 525 \mathrm{~ms}$ case is made clear. While this result is interesting, it may have been expected when one considers the eigenmodes used as inputs (Fig. 10). The decrease in total losses can be easily understood in that the eigenmodes used for the later time case did not extend to the outer flux surfaces as they do for the earlier time simulations, i.e., zero amplitude outside of $\psi^{1 / 2} \approx 0.8$. Having modes which extend to larger radii is a key contributor to global loss since that is where loss boundaries occupy the largest region of phase space in the plasma. As far as decreased losses to FILD, the higher current at the later time serves to move the loss boundaries out to larger radii and away from the localized core modes. The combination of these two effects causes reduced losses at the later time despite having more modes with comparable (actually somewhat larger) mode amplitudes. Both of these effects are shown in Fig. 18. 


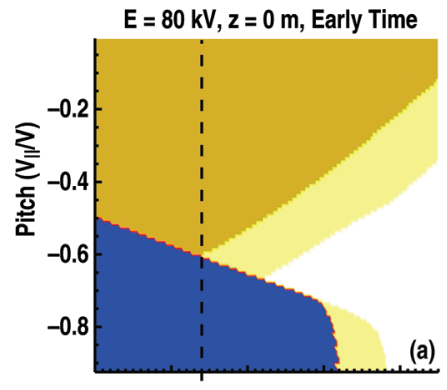

\section{$\mathrm{E}=\mathbf{8 0} \mathrm{kV}, \mathrm{z}=\mathbf{0} \mathrm{m}$, Late Time}
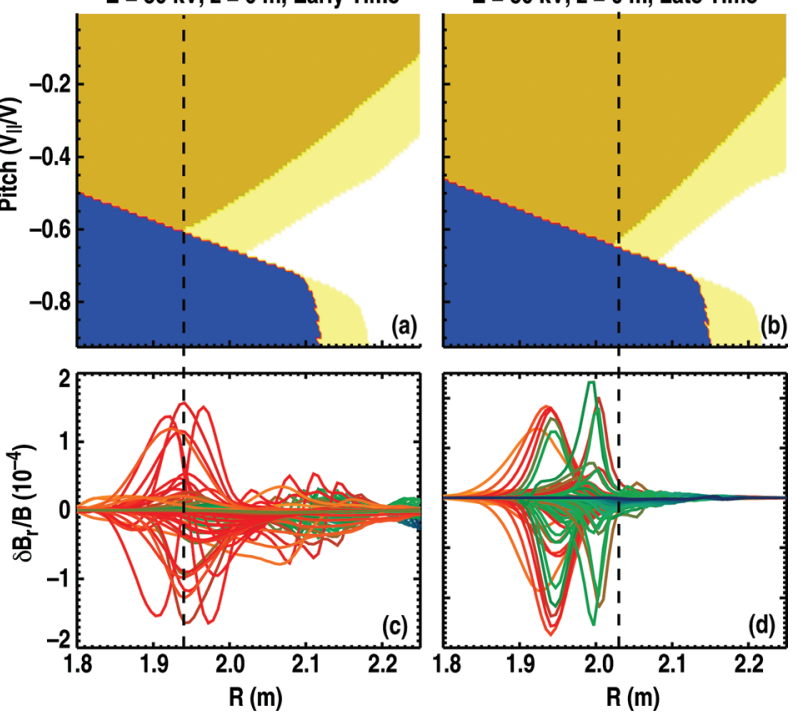

FIG. 18. (Color) Comparison of the loss boundaries at $80 \mathrm{kV}$ along the outboard midplane for the (a) early time case $(t \approx 525 \mathrm{~ms})$ and (b) late time case $(t \approx 725 \mathrm{~ms})$. Yellow and white represent regions from which particles would be lost if launched with energy $80 \mathrm{kV}$ at the corresponding energy and pitch along the outboard midplane. The corresponding eigenmodes along the outboard midplane are shown in (c) and (d), respectively. The dashed vertical line shows the innermost extent of the loss boundaries in each case.

\section{Modeling-coherent loss}

Having seen that the energy, pitch, and long time scale dependence of the measured loss signals can be reproduced with ORBIT modeling, the question remains whether a coherent signature can be extracted from the simulations. The multimode modeling, presented in Sec. III B, was carried out with approximately one million particles spread over a range of energies, pitch angle, and space and as a result lacks sufficient loss statistics to reveal such a feature.

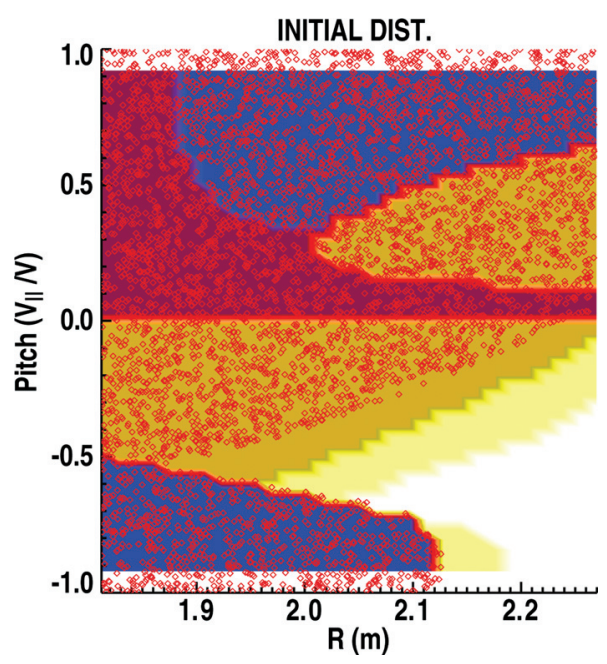

FIG. 19. (Color) Contour shows orbit classifications for $75 \mathrm{keV}$ deuterium ions launched along outboard midplane as a function of major radius and pitch. Blue $=$ passing, gold $=$ trapped, purple $=$ stagnation, yellow and white $=$ intersect vessel wall. Red points indicate random sampling of the 6 million launched particles which were initiated on orbits nominally remaining completely inside of the LCFS.

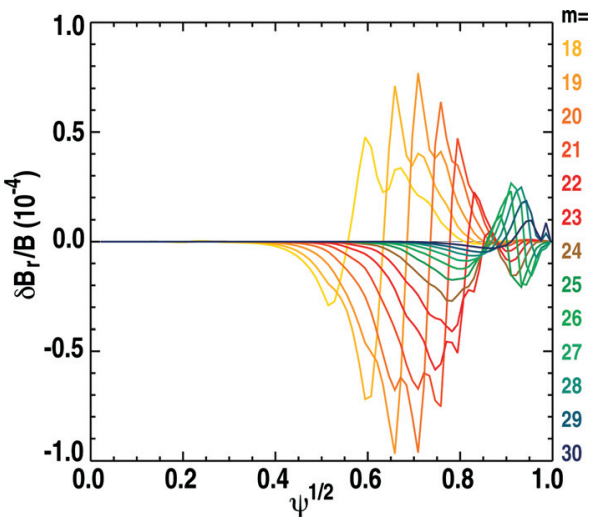

FIG. 20. (Color online) Input radial magnetic field perturbation along outboard midplane. Mode corresponds to an $80 \mathrm{kHz}, n=4$ global TAE. Color coded poloidal mode number in Boozer coordinates of each harmonic is shown to right.

To specifically target the coherent signature, simulations were carried out using six million particles with an initial energy of $75 \mathrm{keV}$ (near the peak of the predicted losses) that were all initially launched along the outboard midplane. These particles were distributed uniformly in major radius and pitch such that nominally all particles were initially on confined orbits with a nominal maximum minor radius of $\psi<1$. Figure 19 shows the orbit classifications as well as a sampling of the initial major radius and pitch of the launched particles. To further simplify the interpretation, a single $f=80 \mathrm{kHz}, n=4 \mathrm{TAE}$ from the previous set of modes at $t \approx 525$ (which were observed to cause coherent loss to FILD) was selected and its amplitude fixed to peak $\delta B_{r} / B \approx 1 \times 10^{-4}$. The outboard midplane structure of the radial magnetic field perturbation for each poloidal harmonic $(m=17-30)$ of this mode is shown in Fig. 20.

The fraction of particles lost versus time with and without the $n=4$ TAE is shown in Fig. 21(a) and the loss rate is shown in Fig. 21(b). Losses in the first $\approx 0.06 \mathrm{~ms}$ are
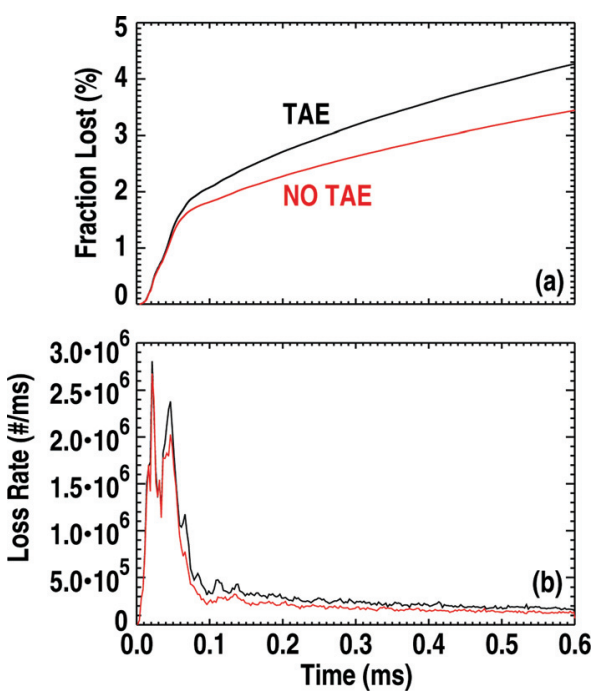

FIG. 21. (Color online) (a) Fraction of lost (hitting LCFS) particles in ORBIT simulation using single $n=4 \mathrm{TAE}$ as well as pitch angle scattering (black) and with only pitch angle scattering (red). (b) Total loss rate in number of simulation particles/ms hitting LCFS. For loss rate, histogramming in time with bin size of $\delta t=0.0025 \mathrm{~ms}$ was used. 

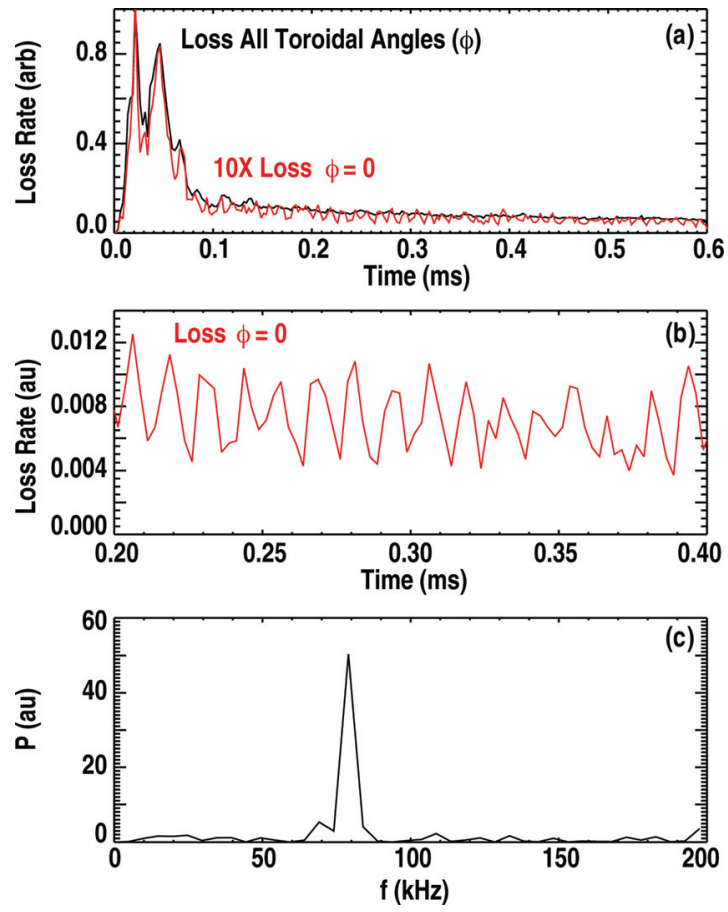

FIG. 22. (Color online) (a) Loss rate of all particles passing through LCFS (black) and $10 \times$ loss rate of particles passing through $\phi=0 \pm 15^{\circ}$ (red). For loss rate, histogramming in time with bin size of $\delta t=0.0025 \mathrm{~ms}$ was used. (b) Enlarged region of panel (a) showing only losses through LCFS at $\phi=0 \pm 15^{\circ}$. (c) Power spectrum of data in panel (b), clearly showing 80 $\mathrm{kHz}$ peak due to $n=4 \mathrm{TAE}$.

dominated by promptly lost particles, then in the "NO TAE" case, losses continue due to pitch angle scattering. Note, even though nominally only confined particles were launched, some prompt losses still exist due to the finite energy and pitch grid used in the COM calculations of the loss boundaries for loading. For the "TAE" case, losses are larger and the difference is directly due to the mode. Figure 22(a) again shows the loss rate versus time with the mode present (black) and overlayed is the losses through a single toroidal slice at $\phi= \pm 15^{\circ}$ (red). For analysis of the time series and extracting a coherent signature, restricting the losses to those occurring at a single toroidal location is necessary and simulates the toroidal localization of the FILD. The losses near $\phi=0$ exhibit a periodic behavior as can be seen more clearly in the shortened time window of Fig. 22(b). The power spectrum of this time series is shown in panel $22 \mathrm{c}$ and a peak at the mode frequency $(f=80 \mathrm{kHz})$ is observed. This indicates a net coherent loss of fast ions through the LCFS due to the $n=4$ TAE.

Similar to the restriction of the losses in toroidal angle, the loss rate versus toroidal angle at a single time can also yield information about the coherent nature of the losses. Figure 23(a) shows the loss rate versus toroidal angle at $t=0.2 \mathrm{~ms}$. The toroidal loss pattern has a clear $n=4$ structure superimposed on a relatively constant dc level. The time evolution of the $n=4$ pattern is shown in Fig. 23(b), where the phase fronts can be seen to propagate around the torus with the mode.

The dc (incoherent) and the coherent $n=4$ structure in Fig. 23(a) are indicative of two distinct types of transport

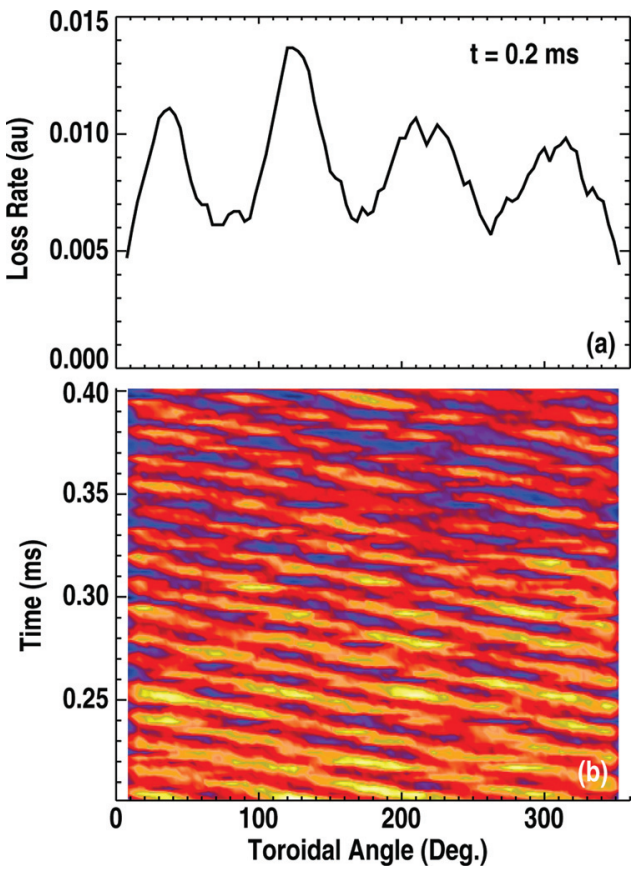

FIG. 23. (Color online) (a) Toroidal distribution of losses through LCFS at simulation time of $t=0.2 \mathrm{~ms}$. (b) Temporal evolution of the toroidal distribution of losses. Overlapping $\delta \phi=30^{\circ}$ windows was used to construct the toroidal distribution in each $\delta t=0.0025 \mathrm{~ms}$ temporal window.

processes. ${ }^{5}$ To further investigate this, the mode amplitude was varied and the level of the incoherent and coherent $n=4$ losses, averaged over several mode cycles near $t=0.2 \mathrm{~ms}$, was recorded. The results of this amplitude scan are shown in Fig. 24. The incoherent losses [Fig. 24(a)] exhibit an amplitude dependence that is quadratic, as one would expect for diffusive type losses. ${ }^{46}$ The initial offset is due to collisions
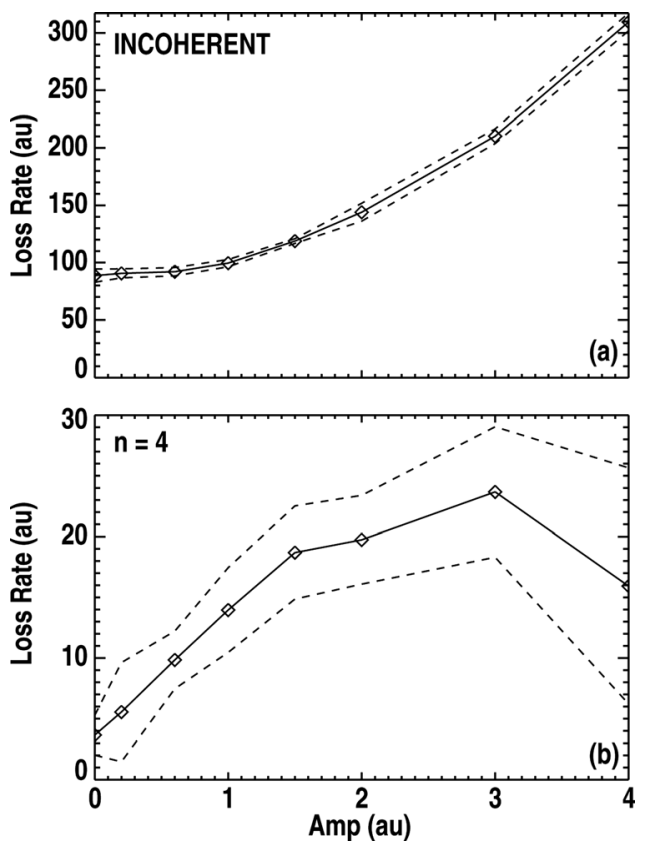

FIG. 24. Dependence of incoherent (a) and coherent $n=4$ (b) loss rates on amplitude scaling factor. Loss rates calculated at simulation time $t \approx 0.2 \mathrm{~ms}$ and averaged over several $80 \mathrm{kHz}$ mode cycles. Dashed lines indicate standard deviation of results from time windows used. 


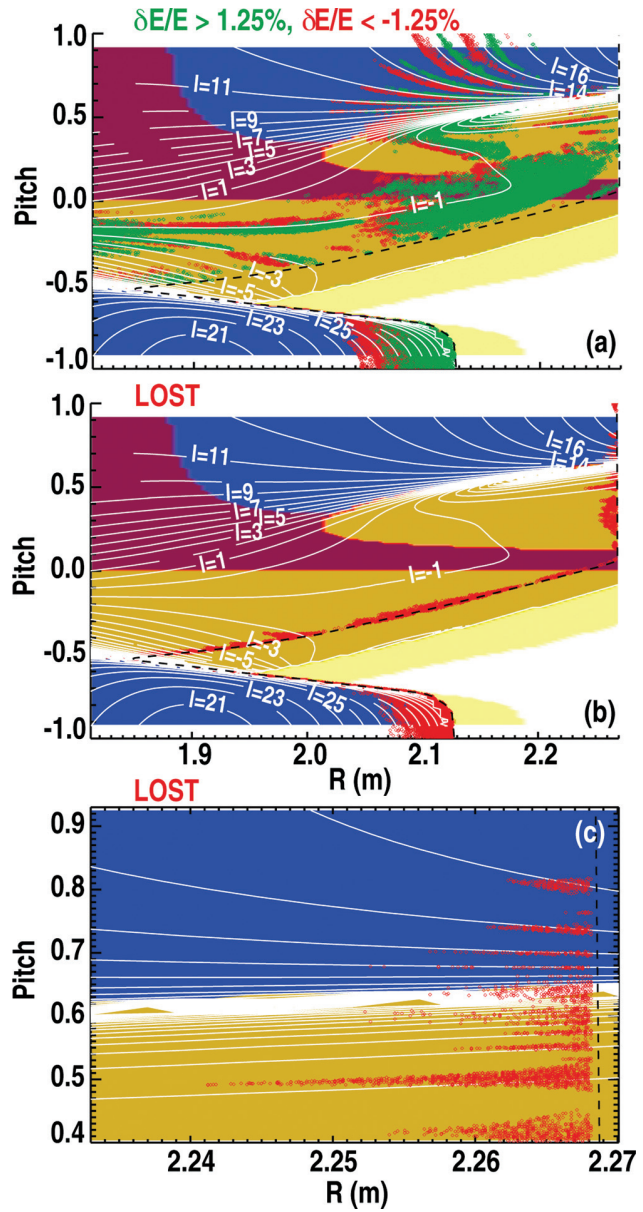

FIG. 25. (Color) Color contours indicate orbit classification of unperturbed orbits: Blue $=$ passing, gold $=$ trapped, purple $=$ stagnation, yellow and white $=$ intersect vessel wall. (a) Initial major radius and pitch of $75 \mathrm{keV}$ ions which had fractional energy increase (green) and decrease (red) greater than $1.25 \%$. Gray contours indicate resonances corresponding to Eq. (1). Dashed black line indicates boundary for particles crossing LCFS. (b) Red points indicate initial major radius and pitch of all nonprompt losses. (c) Zoomed in region of (b) showing losses extending along resonance contours that intersect loss boundary. To clearly show resonance regions, pitch angle scattering was not included in the ORBIT simulation that generated the results shown in this figure.

causing a background of incoherent loss. The coherent loss rate versus amplitude appears to be linear at lower amplitudes then actually decreases at large amplitude. The initial linear dependence on amplitude is indicative of convective losses and is analogous to that observed in Ref. 5. The decreasing at large amplitudes is likely an artifact of the simulation and understood in that at such large mode amplitudes, the resonant particles were expelled so quickly from the system that collisions were not fast enough to replenish the region of phase space available to interact with the mode in the vicinity of the loss boundary.

The losses can be understood better by looking at the details of which particles interact with the mode. Figure 25(a) shows the particle classification diagram as well as the initial pitch and major radius of all particles which gained/ lost (green/red) more than $1.25 \%$ of their initial energy $(75 \mathrm{keV})$. Overlayed on this curve are gray contours corresponding to particles resonances with the $n=4$ TAE. The resonance curves were calculated according to ${ }^{2,41}$

$$
\omega=n \omega_{\phi}-l \omega_{\theta}
$$

where $\omega_{\phi}\left(\omega_{\theta}\right)$ is the toroidal (poloidal) transit frequency for passing particles and the toroidal precession (bounce) frequency for trapped particles. The particle frequencies were calculated using an extended version of the COM based guiding center code which takes into account particle drifts and integrates motion in $(R, z, \phi)$ along constant $\mu$ contours. Immediately, obvious from Fig. 25(a) is that the particles which gain or lose energy occur on opposite sides of a resonance-this is standard Landau trapping where particles slightly below or above resonance gain or lose energy. In the simulation which generated this figure, pitch angle scattering was turned off to make these resonances more clear. This figure accentuates the fact that many resonances play a fundamental role in these discharges, and as a result, much of the fast ion distribution function can interact and exchange energy with the mode. ${ }^{13,16,20,21}$

Figure 25(b) shows all particles which were lost between 0.1 and $0.6 \mathrm{~ms}$ due to the $n=4$ TAE in the simulation (dashed black curve represents bound of particles striking LCFS). Particles are lost from regions along most of the loss boundary, however in regions where resonances intersect or come close to the boundary, more particles appear to be lost. As found in Sec. III B, the largest concentration of lost particles is in the counter passing region. Through the overplotted resonances, it can now be seen that several resonances are very close together in this region and resonance overlap likely occurs, facilitating transport to the loss boundary. Figure 25(c) shows an enlarged region of Fig. 25(b) from the copassing region. Here, the "fingers" of lost particles are clearly seen to extend along the particle resonances and by comparison with Fig. 25(a), it can be seen that these are on the sides of each resonance corresponding to particles that have lost energy to the wave (pitch below for passing and pitch above for trapped).

\section{SUMMARY AND FUTURE WORK}

Measurements of beam driven Alfvén eigenmodes and their impact on both the confined fast ion population as well as ability to cause fast ion loss were presented. Beam power scan experiments on DIII-D showed a transition from classical to $\mathrm{AE}$ dominated fast ion transport at $P_{N B} \approx 2 \mathrm{MW}$, and as previously found, discharges with strong AE activity exhibit a significant deficit in neutron emission relative to classical TRANSP predictions. By keeping beam power constant and delaying injection by $\approx 200-240 \mathrm{~ms}$, during the current ramp, and modifying the current profile, AE activity was reduced or eliminated (a detailed analysis of which will be the subject of future publications) and a significant improvement in neutron emission relative to classical levels was observed. Similarly, experiments in ASDEX Upgrade using early $60 \mathrm{keV}$ neutral beam injection drove multiple unstable RSAEs. Periods of strong RSAE activity were also accompanied by a large (peak $\delta S_{n} / S \approx 60 \%$ ) neutron deficit.

Losses of beam ions modulated at $\mathrm{AE}$ frequencies were observed on DIII-D (Refs. 29, 30) and ASDEX Upgrade ${ }^{14,15}$ using large bandwidth energy and pitch resolving fast ion 
loss scintillator detectors. Modeling of DIII-D loss measurements using the ORBIT guiding center following code to track particles in the presence of NOVA calculated AE structures (validated by comparison to experiment) is able to reproduce the dominant energy, pitch, and temporal evolution of these losses. While loss of both co and counter going fast ions occurs, ORBIT simulations show the dominant loss mechanism observed is the mode induced transition of counter-passing fast ions to lost trapped orbits. In these simulations, approximately $8 \%$ of the confined beam ion population is lost through the LCFS in a full energy slowing down period when using experimental amplitudes.

Modeling also reproduces a coherent signature of $\mathrm{AE}$ induced losses and it was found that these coherent losses scale proportionally with the amplitude (as expected for convective processes); an additional incoherent contribution scales quadratically with the mode amplitude (as expected for losses of a diffusive nature). Interestingly, simplification of the coherent loss problem, such as discussed in Sec. III C, was first carried out using a full-orbit code SPIRAL (Ref. 47) and will be the subject of future publications in which phase of the losses with respect to the mode will be investigated as well as detailed analysis of the full orbit trajectory to FILD. ${ }^{48}$ Future modeling with the SPIRAL code will also benefit from the fact that the equilibrium field is defined to the wall of the vessel, as a result, modeling of losses to FILD can include orbit trajectories which leave and re-enter the plasma continuing to interact with the modes before reaching the FILD.

Extrapolation of loss simulations to ITER and other burning plasma simulations will be carried out in a similar manner to that presented for analysis of DIII-D measurements. For ITER projections, NOVA will be used to calculate eigenmodes of the various predicted scenarios and NOVA-K (Refs. 34, 35) will calculate the unstable eigenmodes. As a validation of the NOVA-K stability calculations and a check on their ability to accurately predict unstable eigenmodes, the same work will include stability calculations for all NOVA eigenmodes in discharge 142111 and a comparison will be made with modes observed to be unstable. Once the unstable eigenmodes are calculated for ITER, both alpha particles and $1 \mathrm{MeV}$ beam ions will then be followed in the presence of the eigenmodes for a large range of amplitudes. To this end, preliminary simulation results for a "Steady State Scenario" plasma indicate that the reduced banana widths in ITER place it in a situation similar to the late time case simulations presented here and very little or no loss occurs even for mode amplitudes as large as $\delta B / B=1 \times 10^{-3}$. An additional interesting question that can be addressed in future work is whether or not core-localized AEs (such as expected) in ITER are able to transport fast ions to large enough radii that they can be subject to additional transport mechanisms created by ripple and TBM induced fields.

\section{ACKNOWLEDGMENTS}

This work was sponsored in part by the US Department of Energy under DE-FC02-04ER54698, SC-G903402, DE-AC02-
99CH11466，DE-FG03-97ER54415，DE-FG02-89ER53296, and DE-FG02-08ER54999. The authors wish to thank both the DIII-D and ASDEX Upgrade teams for making this work possible. In particular, Dr. F. Ryter and Dr. W. Suttrop are gratefully thanked for allowing much earlier beam injection than is typical in ASDEX Upgrade which was critical for obtaining beam driven AEs at high field.

\section{APPENDIX: CALCULATION OF ORBITS OUTSIDE LCFS}

A second code is required to follow particles outside the LCFS since the equilibrium field as implemented in ORBIT is not defined past the LCFS. Fortunately, since the eigenmode amplitudes can be assumed small in this region, simplifications can be made. Outside of the LCFS, a COM based guiding center calculation is performed to follow particles. This calculation assumes conservation of energy, magnetic moment $\left(\mu_{0}\right)$, and toroidal canonical angular momentum $\left(P_{\phi}\right)$, where

$$
\mu_{0}=M_{i} v_{\perp}^{2} / 2 B=\frac{E}{B}\left(1-\chi^{2}\right)
$$

and

$$
P_{\phi}=\psi-\frac{F v_{\|}}{\omega_{c i}}=\psi-\frac{F \chi}{Z e B}\left(2 M_{i} E\right)^{1 / 2}
$$

$F\left(F=B_{t o r} R\right), \psi$ (Poloidal Flux), and $B$ are computed by equilibrium fit (EFIT) on a grid and are independent of the orbit details. Thus, for a given equilibrium, initial position and velocity vector, conservation of energy and toroidal canonical angular momentum determine a pitch everywhere on the $R, z$ grid (some are nonphysical, i.e., $|\chi|>1$ ). The $\chi(R, z)$ grid determines the magnetic moment $\mu(R, z)$ and the $\mu(R, z)=\mu_{0}$ contour passing through the initial position determines uniquely the unperturbed particle orbit in the $R, z$ plane. The $\mu(R, z)=\mu_{0}$ contour path is determined using a simple contour procedure and as such, no integration of the equations of motion is necessary. This approach has been implemented elsewhere ${ }^{49}$ and has also been verified through comparison with full orbit calculations as well as ORBIT calculations inside of the LCFS.

${ }^{1}$ K. L. Wong, Plasma Phys. Controlled Fusion 41, R1 (1999).

${ }^{2}$ W. W. Heidbrink, Phys. Plasmas 15, 055501 (2008).

${ }^{3}$ N. N. Gorelenkov, H. L. Berk, R. Budny, C. Z. Cheng, G.-Y. Fu, W. W. Heidbrink, G. J. Kramer, D. Meade, and R. Nazikian, Nucl. Fusion 43, 594 (2003).

${ }^{4}$ W. W. Heidbrink, M. A. Van Zeeland, M. E. Austin, K. H. Burrell, N. N. Gorelenkov, G. J. Kramer, Y. Luo, M. A. Makowski, G. R. McKee, C. Muscatello, R. Nazikian, E. Ruskov, W. M. Solomon, R. B. White, and Y. Zhu, Nucl. Fusion 48, 084001 (2008).

${ }^{5}$ M. Garcia-Munoz, N. Hicks, R. van Voornveld, I. G. J. Classen, R. Bilato, V. Bobkov, M. Bruedgam, H.-U. Fahrbach, V. Igochine, S. Jaemsae, M. Maraschek, and K. Sassenberg, Phys. Rev. Lett. 104, 185002 (2010).

${ }^{6}$ F. Nabais, V. G. Kiptily, S. D. Pinches, S. E. Sharapov, and JET-EFDA Contributors, Nucl. Fusion 50, 084021 (2010).

${ }^{7}$ E. D. Fredrickson, C. Z. Cheng, D. Darrow, G. Fu, N. N. Gorelenkov, G. Kramer, S. S. Medley, J. Menard, L. Roquemore, D. Stutman, and R. B. White, Phys. Plasmas 10, 2852 (2003).

${ }^{8}$ W. W. Duong, W. W. Heidbrink, T. W. Petrie, R. Lee, R. A. Moyer, and J. G. Watkins, Nucl. Fusion 33, 749 (1993). 
${ }^{9}$ Y. Kusama, H. Kimura, T. Ozeki, M. Saigusa, G. J. Kramer, T. Oikawa, S. Moriyama, M. Nemoto, T. Fujita, K. Tobita, G. Y. Fu, R. Nazikian, and C. Z. Cheng, Nucl. Fusion 38, 1215 (1998).

${ }^{10}$ H. L. Berk, D. N. Borba, B. N. Breizman, S. D. Pinches, and S. E. Sharapov, Phys. Rev. Lett. 87, 185002 (2001).

${ }^{11}$ M. A. Van Zeeland, G. J. Kramer, M. E. Austin, R. L. Boivin, W. W. Heidbrink, M. A. Makowski, G. R. McKee, R. Nazikian, W. M. Solomon, and G. Wang, Phys. Rev. Lett. 97, 135001 (2006).

${ }^{12}$ M. A. Van Zeeland, M. E. Austin, N. N. Gorelenkov, W. W. Heidbrink, G. J. Kramer, M. A. Makowski, G. R. McKee, R. Nazikian, E. Ruskov, and A. D. Turnbull, Phys. Plasmas 14, 056102 (2007).

${ }^{13}$ M. A. Van Zeeland, W. W. Heidbrink, R. Nazikian, M. E. Austin, C. Z. Cheng, M. S. Chu, N. N. Gorelenkov, C. T. Holcomb, A. W. Hyatt, G. J. Kramer, J. Lohr, G. R. McKee, C. C. Petty, R. Prater, W. M. Solomon, and D. A. Spong, Nucl. Fusion 49, 065003 (2009).

${ }^{14}$ M. Garcia-Munoz, I. G. J. Classen, B. Geiger, W. W. Heidbrink, M. A. Van Zeeland, S. Akaslompolo, R. Bilato, V. Bobkov, M. Brambilla, G. D. Conway, S. da Graca, V. Igochine, P. Lauber, N. Luhmann, M. Maraschek, F. Meo, H. Park, M. Schneller, G. Tardini, and the ASDEX Upgrade Team, "Fast Ion Transport Induced by Alfven Eigenmodes in the ASDEX Upgrade Tokamak," Nucl. Fusion (submitted).

${ }^{15}$ M. Garcia-Munoz et al., in Proceedings of 23rd Fusion Energy Conference Daejon, Korea (IAEA, Vienna, 2010).

${ }^{16}$ R. Nazikian, N. N. Gorelenkov, B. Alper, H. L. Berk, D. Borba, R. V. Budny, G. Y. Fu, W. W. Heidbrink, G. J. Kramer, M. A. Makowski, S. D. Pinches, S. E. Sharapov, W. M. Solomon, E. J. Strait, R. B. White, and M. A. Van Zeeland, Phys. Plasmas 15, 056107 (2008).

${ }^{17}$ M. A. Van Zeeland, M. E. Austin, T. N. Carlstrom, T. Deterly, D. K. Finkenthal, C. T. Holcomb, R. J. Jayakumar, G. J. Kramer, M. A. Makowski, G. R. McKee, R. Nazikian, W. A. Peebles, T. L. Rhodes, W. M. Solomon, and E. J. Strait, Nucl. Fusion 46, S880 (2006).

${ }^{18}$ W. W. Heidbrink, N. N. Gorelenkov, Y. Luo, M. A. Van Zeeland, R. B. White, M. E. Austin, K. H. Burrell, G. J. Kramer, M. A. Makowski, G. R. McKee, R. Nazikian, and the DIII-D Team, Phys. Rev. Lett. 99, 245002-1 (2007).

${ }^{19}$ G. Vlad, S. Briguglio, G. Fogaccia, F. Zonca, C. Di Troia, W. W. Heidbrink, M. A. Van Zeeland, A. Bierwage, and X. Wang, Nucl. Fusion 49, 075024 (2009).

${ }^{20}$ R. B. White, N. Gorelenkov, W. W. Heidbrink, and M. A. Van Zeeland, Plasma Phys. Controlled Fusion 52, 045012 (2010).

${ }^{21}$ R. B. White, N. Gorelenkov, W. W. Heidbrink, and M. A. Van Zeeland, Phys. Plasmas 17, 056107 (2010).

${ }^{22}$ R. K. Fisher, D. C. Pace, M. García-Muñoz, W. W. Heidbrink, C. M. Muscatello, M. A. Van Zeeland, and Y. B. Zhu, Rev. Sci. Instrum. 81, $10 \mathrm{D} 307$ (2010).

${ }^{23}$ M. Garcia-Munoz, H. -U. Fahrbach, and H. Zohm, Rev. Sci. Instrum. 80, 053503 (2009).

${ }^{24}$ M. A. Van Zeeland, G. J. Kramer, R. Nazikian, H. L. Berk, T. N. Carlstrom, and W. M. Solomon, Plasma Phys. Control. Fusion 47, L31 (2005).

${ }^{25}$ TRANSP online documentation and instructions as well as Grid Monitor. http://w3.pppl.gov/transp/

${ }^{26}$ I. G. J. Classen, J. E. Boom, W. Suttrop, E. Schmid, B. Tobias, C. W. Domier, N. C. Luhmann, Jr., A. J. H. Donn, R. J. E. Jaspers, P. C. de Vries, H. K. Park, T. Munsat, M. García-Muñoz, and P. A. Schneider, Rev. Sci. Instrum. 81, 10D929-1 (2010).
${ }^{27}$ B. Geiger, M. Garcia-Munoz, W. W. Heidbrink, R. M. McDermott, G. Tardini, R. Dux, R. Fischer, V. Igochine, and the ASDEX Upgrade Team, Plasma Phys. Control. Fusion 53, 065010 (2011).

${ }^{28}$ D. C. Pace, M. García-Muñoz, W. W. Heidbrink, C. M. Muscatello, M. A. Van Zeeland, and Y. B. Zhu, Rev. Sci. Instrum. 81, 10D305 (2010).

${ }^{29}$ D. C. Pace, C. Z. Cheng, R. K. Fisher, M. García-Muñoz, W. W. Heidbrink, Z. Lin, G. R. McKee, M. Murakami, C. M. Muscatello, R. Nazikian, J. M. Park, C. C. Petty, T. L. Rhodes, G. M. Staebler, M. A. Van Zeeland, R. E. Waltz, R. B. White, J. H. Yu, W. Zhang, and Y. B. Zhu, in Proceedings of 23rd Fusion Energy Conference, Daejeon, Korea (IAEA, Vienna, 2010).

${ }^{30}$ D. C. Pace, R. K. Fisher, M. García-Muñoz, W. W. Heidbrink, and M. A. Van Zeeland, "Eigenmode Induced Beam Ion Losses Due to Alfvén Eigenmode Activity in the DIII-D Tokamak," Plasma Phys. Controlled Fusion (to be published).

${ }^{31}$ R. Nazikian, G. Y. Fu, M. E. Austin, H. L. Berk, R. V. Budny, N. N. Gorelenkov, W. W. Heidbrink, C. T. Holcomb, G. J. Kramer, G. R. McKee, M. A. Makowski, W. M. Solomon, M. Shafer, E. J. Strait, and M. A. Van Zeeland, Phys. Rev. Lett. 101, 185001 (2008).

${ }^{32}$ N. N. Gorlenkov, M. A. Van Zeeland, H. L. Berk, N. A. Crocker, D. Darrow, E. Fredrickson, G.-Y. Fu, W. W. Heidbrink, J. Menard, and R. Nazikian, Phys. Plasmas 16, 056107 (2009).

${ }^{33}$ R. B. White and M. S. Chance, Phys. Fluids 27, (1984).

${ }^{34}$ C. Z. Cheng and M. S. Chance, J. Comput. Phys. 71, 124 (1987).

${ }^{35}$ C. Z. Cheng, Phys. Rep. 211, 1 (1992).

${ }^{36}$ E. D. Fredrickson, N. A. Crocker, R. E. Bell, D S. Darrow, N. N. Gorelenkov, G. J. Kramer, S. Kubota, F. M. Levinton, D. Liu, S. S. Medley, M. Podest, K. Tritz, R. B. White, and H. Yuh, Phys. Plasmas 16, 122505 (2009).

${ }^{37}$ M. E. Austin and J. Lohr, Rev. Sci. Instrum. 74, 1457 (2003).

${ }^{38}$ B. Tobias, C. W. Domier, T. Liang, X. Kong, L. Yu, G. S. Yun, H. K. Park, I. G. J Classen, J. E. Boom, A. J. H. Donn, T. Munsat, R. Nazikian, M. Van Zeeland, R. L. Boivin, and N. C. Luhmann, Rev. Sci. Instrum. 81, 10D928 (2010).

${ }^{39}$ B. Tobias, I. G. J. Classen, C. W. Domier, W. W. Heidbrink, N. C. Luhmann, Jr., R. Nazikian, H. K. Park, D. A. Spong, and M. A. Van Zeeland, Phys. Rev. Lett. 106, 075003 (2011).

${ }^{40}$ G. McKee, R. Ashley, R. Durst, R. Fonck, M. Jakubowski, K. Tritz, K. Burrell, C. Greenfield, and J. Robinson, Rev. Sci. Instrum. 70, 913 (1999).

${ }^{41}$ Y. Todo and T. Sato, Phys. Plasmas 5, 1321 (1998).

${ }^{42}$ Y. Todo, H. L. Berk, and B. N. Breizman, Phys. Plasmas 10, 2888 (2003).

${ }^{43}$ S. J. Zweben, R. V. Budny, D. S. Darrow, S. S. Medley, R. Nazikian, B. C. Stratton, E. J. Synakowski. and G. Taylor for the TFTR Group, Nucl. Fusion 40, 91 (2000).

${ }^{44}$ S. J. Zweben, D. S. Darrow, H. W. Herrmann, M. H. Redi, J. F. Schivell, and R. B. White, Nucl. Fusion 35, 1445 (1995).

${ }^{45}$ C. T. Hsu and D. J. Sigmar, Phys. Fluids B 4, 1492 (1992).

${ }^{46}$ D. J. Sigmar, C. T. Hsu, R. White, and C. Z. Cheng, Phys. Fluids B 4, 1506 (1992).

${ }^{47}$ G. J. Kramer et al, in Proceedings of 22nd Fusion Energy Conference, Geneva, Switzerland (IAEA, Vienna, 2008), CD-ROM le IT/P6-3

${ }^{48}$ G. J. Kramer, private communication (2010).

${ }^{49}$ J. Pedersen, Masters Thesis, Niels Bohr Institute, Copenhage University 2004. 